\title{
Stochastic Top K-Subset Bandits with Linear Space and Non-Linear Feedback with Applications to Social Influence Maximization
}

\author{
MRIDUL AGARWAL, VANEET AGGARWAL, and ABHISHEK K. UMRAWAL, \\ Purdue University, USA \\ CHRISTOPHER J. QUINN, Iowa State University, USA
}

\begin{abstract}
There are numerous real-world problems where a user must make decisions under uncertainty. For the problem of influence maximization on a social network, for example, the user must select a set of $K$ influencers who will jointly have a large influence on many users. With the lack of prior knowledge about the diffusion process or even topological information, this problem becomes quite challenging. This problem can be cast as a combinatorial bandit problem, where the user can repeatedly choose a candidate set of $K$ out of $N$ arms at each time, with an aim to achieve an efficient trade-off between exploration and exploitation.

In this work, we present the first combinatorial bandit algorithm for which the only feedback is a nonlinear reward of the selected $K$ arms. No other feedback is needed. In the context of influence maximization, this means no feedback in the form of which nodes or edges were activated needs to be available, just the amount of influence. The novel algorithm we propose, CMAB-SM, is based on a divide-and-conquer strategy It is computationally and storage efficient. Over a time horizon $T$, the proposed algorithm achieves a regret bound of $\tilde{O}\left(K^{\frac{1}{2}} N^{\frac{1}{3}} T^{\frac{2}{3}}\right)$. This bound is sub-linear in all of the parameters: $T, N$, and $K$.

We empirically demonstrate our algorithm's performance using the applications of influence maximization and product cross-selling. For influence maximization, we provide experiments on real-world social networks, showing that the proposed $\mathrm{CMAB}$ algorithm outperforms bandit-specific and social-influence-domainspecific algorithms in terms of empirical run-time and expected influence. For product cross-selling, we also demonstrate that the proposed $\mathrm{CMAB}$ algorithm outperforms considered baselines on synthetic data.
\end{abstract}

CCS Concepts: • Information systems $\rightarrow$ Social networks; • Computing methodologies $\rightarrow$ Planning under uncertainty;

Additional Key Words and Phrases: Combinatorial bandits, social influence maximization, UCB algorithm, $K$-subset selection, regret bounds

This work was presented in part at the 32nd International Conference on Algorithmic Learning Theory (ALT), Mar 2021 [4], which does not include the application to social influence maximization. A preprint of an earlier version of this work is available at [2].

Authors' addresses: M. Agarwal, School of Electrical and Computer Engineering, Purdue University 465, Northwestern Ave, West Lafayette, IN, 47907, USA; email: agarw180@purdue.edu; V. Aggarwal, School of Industrial Engineering, and School of Electrical and Computer Engineering, Purdue University 315 Grant St, West Lafayette, IN, 47907, USA; email: vaneet@purdue.edu; A. K. Umrawal, School of Industrial Engineering, Purdue University, 315 Grant St, West Lafayette, IN, 47907, USA; C. J. Quinn, Department of Computer Science, Iowa State University, 226 Atanasoff Hall, 2434 Osborn Dr, Ames, IA 50011-1090; email: cjquinn@iastate.edu.

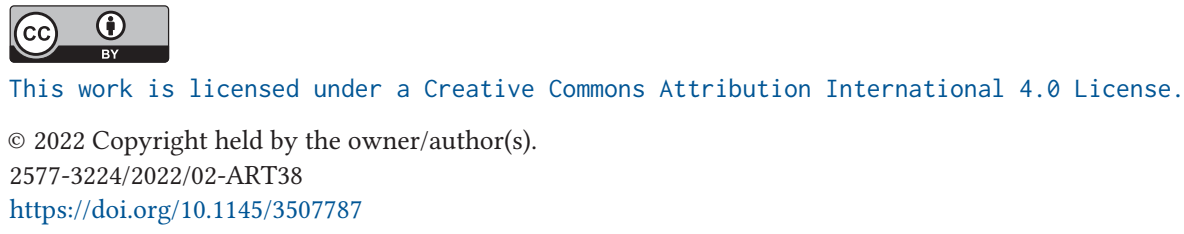


ACM Reference format:

Mridul Agarwal, Vaneet Aggarwal, Abhishek K. Umrawal, and Christopher J. Quinn. 2022. Stochastic Top $K$-Subset Bandits with Linear Space and Non-Linear Feedback with Applications to Social Influence Maximization. ACM/IMS Trans. Data Sci. 2, 4, Article 38 (February 2022), 39 pages.

https://doi.org/10.1145/3507787

\section{INTRODUCTION}

There are numerous settings where agents make decisions sequentially within an unknown, stochastic environment, and each decision involves choosing a subsets of options. Two important examples are social influence maximization and product cross-selling.

In lieu of traditional media advertising, a company may repeatedly select a set of social media "influencers" to sponsor, wanting those influencers to inspire their followers, and followers of those followers, to discuss and post about the product. The problem of sequentially selecting a set of influencers to sponsor, known as social influence maximization [15], can be challenging. The dynamics of how influence spreads among social network users may be complex and difficult to accurately model, with privacy settings limiting visibility, unknown user preferences, overlapping audiences, and interactions between multiple social networks.

Researchers have designed algorithms for social influence maximization based on algorithms for combinatorial multi-armed bandits with semi-bandit feedback. Those algorithms assume knowledge of the network topology [20,30], influence propagation traces [20], node-level feedback [45], edge-level feedback [40], etc. However, such feedback may be unavailable due to privacy settings. We consider the case when such feedback is not available and model the problem as a combinatorial multi-armed bandit with full-bandit feedback, i.e. no knowledge of the underlying social network or diffusion process except the number of posts.

The problem maximal-profit item selection with cross-selling [42], which is defined as follows: "if selling items together is more profitable than selling them individually then which two or more items should be sold together". The problem is about selecting a subset of items which can give the maximal profit with the consideration of cross-selling in an online manner.

Other motivating applications include erasure-coded storage where $K$ out of $N$ servers are picked to serve a content request [43], and daily advertising campaigns involving a set of $K$ subcampaigns [36, 44].

All the applications discussed above can be modeled as combinatorial multi-armed bandit (CMAB) problems. There are $N$ options or "arms" available at each time. At each time, the agent can choose up to $K$ arms and will receive a reward depending on the chosen subset of arms. The goal of the agent is to obtain as much reward as possible over the time horizon. As in the traditional MAB problem, where the agent can only select $K=1 \mathrm{arm}$, the agent may need to balance "exploration," that is picking a subset of $K$ arms to learn about the subset's reward distribution, and "exploitation," that is picking a subset of $K$ arms that currently seems the best, despite the agent having uncertainty about each subset.

Unlike the traditional setting, however, when the agent can choose any subset of $K>1$ arms out of all $\left(\begin{array}{l}n \\ k\end{array}\right)$ such subsets, it may be impossible to even try each subset once. Furthermore, when the subset's reward is an unknown, non-linear function of the individual arms' rewards, it may be impossible to infer each arms' individual performance. Previous combinatorial MAB algorithms that assume a linear function or require additional feedback in the form of each individual arms' performance $[11,35]$ will not work for this setting.

In this paper, we propose an efficient algorithm, CMAB-SM to solve the CMAB problem where the reward is a non-linear function of individual arm rewards and no additional feedback is 
available. To our knowledge, this is the first paper to do so. CMAB-SM achieves a regret bound of $\widetilde{O}\left(K^{\frac{1}{2}} N^{\frac{1}{3}} T^{\frac{2}{3}}\right)$. Our proposed algorithm only requires $O(N)$ storage complexity and $\tilde{O}(K)$ per-round time complexity. ${ }^{1}$

\subsection{Our Contributions}

We now summarize the main contributions of this paper:

(1) We propose CMAB-SM, the first, efficient algorithm for the CMAB problem for non-linear "full-bandit" feedback (i.e. no extra feedback for individual arms).

(2) We prove that over a horizon $T$, CMAB-SM achieves a regret of $\widetilde{O}\left(K^{\frac{1}{2}} N^{\frac{1}{3}} T^{\frac{2}{3}}\right)$, which is $s u b$ linear with respect to each parameter.

(3) We prove that CMAB-SM has $O(N)$ space complexity and $O(T K \log K)$ time complexity.

(4) We evaluate CMAB-SMś performance empirically in multiple problems, including influence maximization. CMAB-SM outperforms the $\epsilon$-CD algorithm (a domain-specific algorithm based on the credit distribution model [20]).

(5) Lastly, the design and analysis of CMAB-SM uses the theory of stochastic dominance to order the arms without needed to estimate individual arm distributions. This technique obviates the need of additional feedback. This method may be of independent interest for MAB problems.

\subsection{Key Techniques}

We now summarize the method and proof techniques used. CMAB-SM divides all $N$ arms into groups of $K+1$ arms, such that each group contains only $K+1$ actions as there are $K$ arms to choose from $K+1$ arms. Since choosing $K$ out of $K+1$ is equivalent to removing 1 out of $K+1$, there is a one-to-one mapping between arms and actions in a group. We sort the actions (and the corresponding arms) in each group which requires time steps of polynomial order in $K$. We then merge those groups one by one and obtain the best $K$ arms.

For the analysis, we assume certain properties on the reward distributions of the different arms, and on the function of rewards of individual arms. More precisely, we use the theory of stochastic dominance to differentiate between cumulative distribution functions. The reward distributions of different arms are assumed to dominate or be dominated by each other. Further, the non-linear function is assumed to be symmetric in the rewards obtained from each arm, and the mean of the function is assumed to be continuous (in terms of dominated inputs). These properties are satisfied in case of a few reward distributions (e.g., Bernoulli rewards), and few non-linear functions (e.g., maximum).

\subsection{Related Work}

Combinatorial Bandits have been studied where the agent chooses $K$ of the $N$ arms in each round $[1,5,10,12-14]$. In these works, it is assumed that the reward function in each round is linear in the different arms. They consider a setting where at time $t$, the agent selects an arm $x_{t} \in D_{t}$ and observes a reward $\theta^{T} x_{t}$, where $D_{t} \subset \mathbb{R}^{K}$ is the decision set and $\theta \in \mathbb{R}^{K}$ is a constant vector. Due to this linear function, the problem is also called online linear optimization. The algorithms proposed in these works use the linearity of the reward function to estimate rewards of individual arms and achieve a regret of $O(\sqrt{T})$. Weights are then assigned to each of the $\left(\begin{array}{l}N \\ K\end{array}\right)$ actions to decide the action in the next round; such approaches are not computationally efficient for large $N$ as $\left|D_{t}\right|=\left(\begin{array}{l}N \\ K\end{array}\right)$. The work of [34] reduces the space complexity at the cost of regret bounds. However, they still

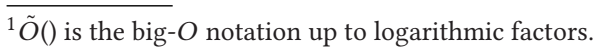


loop over all the arms and the regret bound becomes exponential in $K$. For a linear function, such as sum of rewards, we can construct arms which is a binary $K$-sparse vector of length $N$. Such a setting has $N$ unknown variables, and those unknowns could be obtained using least squares as done by [12] or regularized least squares [1]. We consider non-linear functions for which the expected rewards of individual arms could not be obtained using least squares solution.

$[16,22,33]$ studied the problem of generalized linear models (GLM) where reward $r_{t}$ is a function $(f(z): \mathbb{R} \rightarrow \mathbb{R})$ of $z=\theta^{T} x_{t}$. Generalized linear models assume the distribution of bandit reward $r_{t}$ belong to a cannonical exponential family. The exponential distribution allows the use log-likelihood maximization to obtain estimates of arm parameters which increase the likelihood of observed rewards. Generalized Linear Models assume that the expected reward of the arm played is a non linear function of the linear combination of features of the action played with a fixed parameter. This is different than our setup where we assume the reward of the action played is a non linear function of individual realization of rewards of each arm.

GLM (and linear) models have a long and rich history across many disciplines such as finding a target item among multiple options [29]. GLMs also have many interesting theoretical and statistical properties. But there are settings where GLMs do not accurately model rewards. For example, in the case where multiple arms are selected and the joint reward is the maximum of individual rewards, the joint rewards is not a linear combination of the individual arm rewards.

[27] provides an UCB style algorithm for matroid bandits, where the agent selects a maximal independent set of rank $K$ to maximize sum of rewards of each arm. They assume rewards of each of the $K$ arms is also observed in each round. Such a setup, where the rewards of each of the $K$ arms is also available to agent, is referred to as a semi-bandit problem. [17] also considered the problem of semi-bandits for the problem of maximum weighted matching for cognitive radio applications. [28] showed that the UCB algorithm provides a tight regret bound for the semi-bandit combinatorial bandit problem with linear reward function. The authors of [11] considered the combinatorial semi-bandit problem with non-linear rewards using a UCB style analysis. The authors of [35] assumed the combinatorial bandit problem with non-linear reward function and feedback, where the feedback is a linear combination of rewards of the $K$ arms. Such feedback of a linear function of rewards allows for the recovery of individual rewards. In contrast to prior works, this paper does not consider the availability of individual arm rewards or a linear feedback. With only aggregate, non-linear feedback, it might not be possible to obtain the exact values of the rewards of base arms.

\subsection{Organization}

The rest of the paper is organized as follows. In Section 2, we provide the model under consideration and the assumptions that are taken for the analysis. Section 3 presents the proposed algorithm, CMAB-SM. The main result is provided in Section 4. Section 5 illustrates our results on a synthetic example for the cross-selling application. Further evaluations on synthetic problems are provided in the Appendix. In section 6, we present the results for the application to Influence Maximization and compare the proposed approach with some standard domain-specific methods. Section 7 presents the conclusions with directions for future work.

\section{PROBLEM FORMULATION AND ASSUMPTIONS}

\subsection{Problem Setup}

We now describe the stochastic combinatorial multi-armed bandit problem we consider. There are $N$ "arms" labeled as $i \in[N]=\{1,2, \ldots, N\}$. Each time an arm is chosen or "played," there is a reward. Let $X_{i, t} \in[0,1]$ be a random variable denoting the reward of the $i^{t h}$ arm, at time-step $t$ (also referred to as time $t$ ). We assume that $X_{i, t}$ are independent across time and arms, and for 
any arm the distribution is identical at all times. Also, if some analysis is independent of the time variable, we will drop the subscript $t$ and write only $X_{i}$. The rewards could be discrete valued, continuous valued, or mixed.

At each time instant, the agent chooses an action $\mathbf{a}=\left(a_{1}, a_{2}, \ldots, a_{K}\right)$ which is a $K$-tuple of arms. Let $\mathcal{A}=\left\{\mathbf{a} \in[N]^{K} \mid \mathbf{a}(i) \neq \mathbf{a}(j) \forall i, j: 1 \leq i<j \leq K\right\}$ be the set of all such actions which can be constructed using $N$ arms. Thus the cardinality of $\mathcal{A}$ is $\left(\begin{array}{l}N \\ K\end{array}\right)$. We denote the action played at time $t$ as $\mathbf{a}_{t} \in \mathcal{A}$. For an action $\mathbf{a}=\left(a_{1}, a_{2}, \ldots, a_{K}\right)$ let $\mathbf{d}_{\mathrm{a}, t}=\left(X_{a_{1}, t}, X_{a_{2}, t}, \ldots, X_{a_{K}, t}\right) \in[0,1]^{K}$ denote the column vector of arm rewards at time $t$ from action a. The reward $r_{\mathbf{a}}(t)$ of action a at time $t$ is a bounded function $f:[0,1]^{K} \rightarrow[0,1]$ of the rewards from the arms chosen in that action,

$$
r_{\mathrm{a}}(t)=f\left(\mathbf{d}_{\mathrm{a}, t}\right) .
$$

Later in the text, we will skip index $t$ for brevity, where it is unambiguous. If at time $t$, action $\mathbf{a}_{t}$ is played, $\mathbf{d}_{\mathrm{a}_{t}, t}$ will be simplified to $\mathbf{d}_{\mathrm{a}_{t}}$. Also, if we analyze behavior for action a such that its reward vector is independent and identically distributed across time, we will drop the subscript and use only $\mathbf{d}_{\mathrm{a}}$ for brevity.

In many practical systems, the real reward is a non-linear function of noisy reward instead of a non-linear function of expected rewards plus noise. For example, consider a distributed system. A job may be forked into multiple parallel tasks. The completion time of the job depends on the maximum time taken to finish any of the task. Hence, the completion time of the job is a nonlinear function of the completion time of the sub-tasks. Another example for non-linear function of individual rewards is item selection in cross selling. In cross-selling, the total profit of the seller is the sum of individual profits plus an additional advantage made from the combined transaction. The additional advantage can be modelled as a quadratic term of the individual items sold [42].

For a linear case, the two cases are equivalent as

$$
r_{\mathrm{a}}(t)=f\left(\mathbf{d}_{\mathrm{a}_{t}}\right)=\theta^{T} \mathbf{d}_{\mathbf{a}_{t}}=\theta^{T}\left(\mathbb{E}\left[\mathbf{d}_{\mathrm{a}_{t}}\right]+\eta_{t}\right)=\theta^{T} \mathbb{E}\left[\mathbf{d}_{\mathrm{a}_{t}}\right]+\theta^{T} \eta_{t}=f\left(\mathbb{E}\left[\mathbf{d}_{\mathrm{a}_{t}}\right]\right)+\epsilon_{t}
$$

where $\epsilon_{t}$, and $\eta_{t}$ are noise terms.

For a non-linear case, this formulation does not simply reduces to function of expected rewards plus noise. However, we can still write the reward as the expected bandit reward plus noise $\epsilon_{t}$, or

$$
r_{t}=\mathbb{E}\left[f\left(\mathbf{d}_{\mathbf{a}_{t}}\right)\right]+\epsilon_{t}
$$

Similar to the work of [6], we aim to maximize the expected reward for the actions selected over time. Further, we intend to improve the finite time regret bounds for the same. We denote the expected reward of any action $\mathbf{a} \in \mathcal{A}$ as $\mu_{\mathrm{a}}$, or $\mu_{\mathrm{a}}=\mathbb{E}\left[r_{\mathrm{a}}(t)\right]$ for all $t \in\{1,2 \ldots, T\}$.

We assume that there is an action $\mathbf{a}^{*}$ for which the expected reward $\mu_{\mathrm{a}^{*}}$ is highest among all actions a $\in \mathcal{A}$,

$$
\mathbf{a}^{*}=\arg \max _{\mathbf{a} \in \mathcal{A}} \mu_{\mathbf{a}}
$$

We refer to this action $\mathrm{a}^{*}$ as "optimal." Given an optimal action, regret for an action at time $t$ can be defined as follows.

Definition 2.1 (Regret). The regret of an action $\mathrm{a}_{t}$ at time $t$ is defined as the difference between the reward obtained by the optimal action and the reward obtained by $\mathbf{a}_{t}$, or

$$
R(t)=r_{\mathrm{a}^{*}}(t)-r_{\mathrm{a}_{t}}(t)
$$

The objective is to minimize the expected regret accumulated during the entire time horizon,

$$
W(T)=\mathbb{E}_{\mathbf{a}_{1}, r_{\mathrm{a}_{1}}(1), \ldots, \mathbf{a}_{T}, r_{\mathrm{a}_{T}}(T)}\left[\sum_{t=1}^{T} R(t)\right]
$$




\subsection{Assumptions}

We now discuss the technical assumptions which are required to prove the regret bounds for the CMAB-SM algorithm. We note that many of the assumptions are not required for the algorithm to work, only to prove the guarantees.

The function $f$ is assumed to be symmetric, so that the ordering within the tuple does not matter. In other words, the rewards for an action is symmetric in its constituent arms. This assumption is true for certain problem settings where the ordering among the individual arms is not important, like the maximum of rewards, or the sum of rewards of the individual arms. This assumption is given as follows.

Assumption 1 (Symmetry). $f$ is a symmetric function of the rewards obtained by the constituent arms. More precisely, let $\Pi(\mathbf{d})$, be an arbitrary permutation of $\mathbf{d}$. Then, the reward observed will be identical for both $\Pi(\mathbf{d})$ and $\mathbf{d}$, or

$$
f(\mathbf{d})=f(\Pi(\mathbf{d}))
$$

In the rest of the text, we denote $\Pi(\cdot)$ as a permutation, where $\Pi(y)$ is one of the possible permutations of the vector $\mathbf{y}$. We now define gap $\Delta$ between two actions as follows.

Definition 2.2 (Gap). The Gap $\Delta_{\mathbf{a}_{1}, \mathbf{a}_{2}}$ between any two actions $\mathbf{a}_{1}, \mathbf{a}_{2} \in \mathcal{A}$ is defined as the difference of expected rewards of the actions, or

$$
\Delta_{\mathrm{a}_{1}, \mathrm{a}_{2}}=\mu_{\mathrm{a}_{1}}-\mu_{\mathrm{a}_{2}}=\mathbb{E}\left[f\left(\mathbf{d}_{\mathrm{a}_{1}}\right)\right]-\mathbb{E}\left[f\left(\mathbf{d}_{\mathrm{a}_{2}}\right)\right]
$$

We assume that there is an optimal action $\mathrm{a}^{*}$ for which the expected reward is highest among all actions $\mathbf{a} \in \mathcal{A}$. We denote the reward of the optimal action by $\mu_{\mathrm{a}^{*}}$. The Gap of an action $\mathrm{a} \in \mathcal{A}$ with respect to the optimal action is simply written as $\Delta_{\mathbf{a}}$, or, $\Delta_{\mathbf{a}}=\Delta_{\mathbf{a}^{*} \text {,a }}$

Remark 1. Using linearity of expectation it can be seen that, $\mathbb{E}[R(t)]=\Delta_{\mathbf{a}_{t}}$

From Remark 1, the expected regret accumulated during the entire time horizon can be written as,

$$
W(T)=\mathbb{E}\left[\sum_{t=1}^{T} R(t)\right]=\mathbb{E}\left[\sum_{t=1}^{T} \Delta_{\mathbf{a}_{t}}\right]
$$

We define the maximum regret of all possible actions as $R_{\max }=\max _{\mathbf{a} \in \mathcal{A}} \Delta_{\mathbf{a}}$.

We now use the concept of stochastic dominance to order two arms. Assume that there exists a first-order stochastic dominance between any two arms which is defined as follows.

Definition 2.3 (First-Order Stochastic Dominance). A random variable $X$ has first-order stochastic dominance (FSD) over another random variable $Y$ ( or $X>Y$ ), if for any outcome $x$, $X$ gives at least as high a probability of receiving at least $x$ as does $Y$, and for some $x, X$ gives a higher probability of receiving at least $x$,

$$
\begin{gathered}
X>Y \Leftrightarrow P(X \geq x) \geq P(Y \geq x) \forall x \in \mathbb{R}, \\
P(X \geq x)>P(Y \geq x) \text { for some } x \in \mathbb{R} .
\end{gathered}
$$

Assumption 2 (FSD Between ARms). There exists a dominance ordering between all the arms, which is defined using FSD. In other words, for each pair of arms $i$ and $j$, either $X_{i}>X_{j}$ or $X_{j}>X_{j}$.

The FSD implies second order stochastic dominance, which indicates that the mean of the dominating random variable is at least as much as the mean of the dominated random variable [7, 21]. This is summarized in the following lemma. 
Lemma 2.4 ([7, 21]). If a random variable $X$ has FSD over another random variable $Y$ (or, $X>Y$ ), then the expected value of $X$ is at least the expected value of $Y$, or

$$
\mathbb{E}[X] \geq \mathbb{E}[Y]
$$

Remark 2. From Lemma 2.4, we note that if $\operatorname{arm} i$ dominates $\operatorname{arm} j$, then the mean reward for arm $i$ is strictly greater than that of $\operatorname{arm} j$. Thus, under Assumption 2,

$$
\mathbb{E}\left[X_{i}\right] \neq \mathbb{E}\left[X_{j}\right], \forall i, j \in\{1, \ldots, N\}
$$

Such strict dominance exists for Bernoulli and exponential reward distribution functions.

Since we can construct a new action by changing the arms of an existing action, we define the replacement function $h(\cdot, \cdot, \cdot)$ which changes an element $i$ of a given reward vector $\mathbf{d}$ (where each entry in the reward vector is a random variable with the distribution of the corresponding arms).

Definition 2.5 (Replacement Function). The replacement function $h(\cdot)$ is defined as a function on $\mathbb{R}^{K+2}$, which replaces the $i^{\text {th }}$ element of vector $\mathbf{d}$ with $x$, or

$$
h(\mathbf{d}, i, x)=(\mathbf{d}(1), \ldots, \mathbf{d}(i-1), x, \mathbf{d}(i+1), \ldots, \mathbf{d}(K)) .
$$

For a random variable $X, h(\mathbf{d}, i, X)$ is also a random variable. We also assume that the expected reward of an action is strictly increasing function of the rewards obtained by the individual arms.

Assumption 3 (Strictly InCREASING). $f(\cdot)$ is element-wise, strictly increasing function of the individual rewards obtained by the constituent arms. More precisely,

$$
f(h(\mathbf{d}, i, x))>f(h(\mathbf{d}, i, y)) \forall x>y ; x, y \in[0,1] \forall \mathbf{d} \in[0,1]^{K}
$$

Even though we assume strictly increasing function, the analysis also holds for strictly decreasing function $f$ by transforming the reward function as $f_{n}(\mathbf{d})=1-f(\mathbf{d})$. In order to compare the distance between individual reward vectors from two different actions, we need to find the difference in the two individual reward vectors up to a permutation, since the reward function is permutation invariant. With this distance metric in mind, we assume that $f(\cdot)$ is Lipschitz continuous (in an expected sense), which is formally described in the following.

Assumption 4 (Continuity of Expected Rewards). The expected value of $f(\cdot)$ is Lipschitz continuous with respect to the expected value of the rewards obtained by the individual arms, meaning

$$
\left|\mathbb{E}\left[f\left(\mathbf{d}_{1}\right)\right]-\mathbb{E}\left[f\left(\mathbf{d}_{2}\right)\right]\right| \leq U_{1} \min _{\Pi}\left\|\mathbb{E}\left[\mathbf{d}_{1}\right]-\Pi\left(\mathbb{E}\left[\mathbf{d}_{2}\right]\right)\right\|_{2}
$$

for any given random vectors $\mathbf{d}_{1}$ and $\mathbf{d}_{2}$ and for some $U_{1}<\infty$, where $\Pi$ is minimized over all permutations of $\{1, \ldots, K\}$.

Corollary 2.6. Assumption 4 also implies

$$
|\mathbb{E}[f(h(\mathbf{d}, i, X))]-\mathbb{E}[f(h(\mathbf{d}, i, Y))]| \leq U_{1}|\mathbb{E}[X]-\mathbb{E}[Y]|
$$

for any given random vector $\mathbf{d}$ and any $i \in\{1, \ldots, K\}$.

We further assume a lower bound in (13) as formally stated in the following assumption.

Assumption 5 (CONTINUity of Individual Expected RewArds). We also assume that the continuity given in (13) also has a similar lower bound. More precisely, there is a $U_{2}<\infty$ such that

$$
|\mathbb{E}[X]-\mathbb{E}[Y]| \leq U_{2}|(\mathbb{E}[f(h(\mathbf{d}, i, X))]-\mathbb{E}[f(h(\mathbf{d}, i, Y))])|
$$

for any given random vectors $\mathbf{d}$ and any $i \in\{1, \ldots, K\}$. 
Assumption 5 holds for many well behaved functions in practical scenarios. For example, $f(\cdot)=$ $\max (\cdot)$ with Bernoulli rewards for individual arms, ${ }^{2} f(\cdot)=$ sum of individual rewards, or $f(\cdot)=$ concave utility function of sum of individual rewards.

Corollary 2.7. Combining Corollary 2.6 and Assumption 5 and defining $U=\max \left(U_{1}, U_{2}\right)$, we have

$$
\begin{aligned}
\frac{1}{U}|\mathbb{E}[X]-\mathbb{E}[Y]| & \leq|(\mathbb{E}[f(h(\mathbf{d}, i, X))]-\mathbb{E}[f(h(\mathbf{d}, i, Y))])| \\
& \leq U|\mathbb{E}[X]-\mathbb{E}[Y]|,
\end{aligned}
$$

for any given random vector $\mathbf{d}$ and any $i \in\{1, \ldots, K\}$.

We note that linear bandits become a special case of the assumptions we considered for $U=\sqrt{K}$.

\section{PROPOSED ALGORITHM}

The proposed algorithm, called CMAB-SM, is an explore then exploit strategy which aims to minimize the expected regret, be computationally efficient, and have a storage complexity which is linear with $N$ and independent of K. CMAB-SM, described in Algorithm 1, utilizes the fact that for CMAB problem, choosing $K$ arms from a set of $K+1$ arms has $K+1$ actions thus making the problem solvable using the standard MAB approach. If $N=K+1$, then the complexity is $\left(\begin{array}{c}K+1 \\ K\end{array}\right)=K+1$, and only $K+1$ actions needs to be optimized. The intuition to this approach follows closely to the Merge Sort algorithm, where the elements of the array are noisy. Hence, to obtain a better estimate of the array elements, we sample them repeatedly.

We construct a group $G$ which is a vector of length $K+1$ consisting of arm indices, or $G \in[N]^{K+1}$. Then, we can construct $K+1$ actions, each action using all but one entries in the group $G$.

Let $\mathbf{a}_{-i}^{G}$ be an action in group $G$ with $G(i)^{t h}$ arm left out, where $G(i), i \in\{1, \ldots, K+1\}$, is the $i^{t h}$ entry of the group.

With $X_{G(i)}$ denoting the reward of arm $G(i)$, the individual reward vector $\mathbf{d}_{\mathbf{a}_{-i}^{G}}$ with the action $\mathbf{a}_{-i}^{G}$ is

$$
\mathbf{d}_{\mathbf{a}_{-i}^{G}}=\left(X_{G(1)}, \ldots, X_{G(i-1)}, X_{G(i+1)}, \ldots, X_{G(K+1)}\right) .
$$

The (random) reward obtained at any time with this action is $r_{\mathbf{a}_{-i}^{G}}=f\left(\mathbf{d}_{\mathrm{a}_{-i}^{G}}\right)$, with a mean reward of $\mu_{\mathrm{a}_{-i}^{G}}=\mathbb{E}\left[f\left(\mathbf{d}_{\mathrm{a}_{-i}^{G}}\right)\right]$. The next result shows that an ordering on $\left(\begin{array}{c}K+1 \\ K\end{array}\right)$ actions made using $K+1$ arms gives an ordering on $K+1$ arms under the considered assumptions.

Lemma 3.1. An ordering on $\left(\begin{array}{c}K+1 \\ K\end{array}\right)$ actions, in group $G$, made using $K+1$ arms gives an ordering on $K+1$ arms. In other words, if an ordering exists between actions $\mathbf{a}_{-i}^{G}$ and $\mathbf{a}_{-j}^{G}$, then an ordering exists between arms $G(i)$ and $G(j)$. More precisely,

$$
\mu_{\mathrm{a}_{-i}^{G}}>\mu_{\mathrm{a}_{-j}^{G}} \Rightarrow \mathbb{E}\left[X_{G(i)}\right]<\mathbb{E}\left[X_{G(j)}\right]
$$

Proof. (Outline): If we have two actions made from group $G$ by excluding arm $G(i)$ and $\operatorname{arm} G(j)$ respectively, then the arm with higher reward will increase the joint reward $f$ as $f$ is an increasing function. The detailed proof is provided in Appendix B.

\footnotetext{
${ }^{2}$ We note that maximum function, in general, does not satisfy Assumption 5. However, if an arm exists such that its rewards are always higher compared to other arms, the agent can place any other arm among the $K-1$ arms, without incurring any regret.
} 
CMAB-SM divides all $N$ arms into groups of $K+1$ arms arbitrarily. Each group now contains only $K+1$ actions. If the last group contains less than $K+1$ arms (if $N \bmod (K+1)>0)$, arms from other groups are added (repeated) to have $K+1$ arms in the last group. CMAB-SM then picks the first group of $K+1 \mathrm{arms}$ and orders the arms in the group using SORT subroutine. Using this subroutine, the $K+1 \mathrm{arms}$ in the group are ordered with respect to expected individual rewards. We also consider $G^{*}$ as the best $K$ arms seen so far, which are the top $K$ arms in $G_{1}$. It later proceeds in $k \in\left\{2, \ldots, \frac{N}{K+1}\right\}$ rounds. ${ }^{3}$ In $k^{t h}$ round it performs SORT on $G_{k}$ and merge $G_{k}$ and $G^{*}$ using MERGE subroutine to obtain a new $G^{*}$. The SORT subroutine orders the $K+1$ arms in $G_{k}$. The MERGE subroutine takes the best $K$ arms before this round, $G^{*}$; and the best $K$ arms from the SORT subroutine on $G_{k}$ and merges them to find the best $K$ arms seen so far and saves them as $G^{*}$. This is then inputted to the next value of $k$ to merge with other groups.

At the end of $\left(\frac{N}{K+1}\right)^{t h}$ round, we would have played all arms in each group and merged them, thus resulting in an optimal action which maximizes the expected reward for the remaining time slots. Apart from the sort and merge scheme, we also use a hyperparameter $\lambda$ in our algorithm. $\lambda$ denotes the minimum gap the agent can resolve between any two arms. We choose the value of hyperparameter which minimizes the regret from the CMAB-SM algorithm. Following the regret analysis, the hyperparameter is specified in (30). If there is any the gap between arm $i$ and arm $j$, the algorithm cannot determine which arm is better with high probability and selects the arm with higher sample mean as the better arm. This behaviour is common in both SORT and MERGE subroutines. We now describe the algorithms used in CMAB-SM which are SORT and MERGE subroutines in detail.

\subsection{SORT}

The SORT subroutine is given in Algorithm 2. In this subroutine, we play $K+1$ actions formed from $K+1$ arms in a group $G$, each action corresponding to one left out arm. The subroutine proceeds in rounds similar to UCB algorithm by [6]. By the end of round $r$, each action is played $n_{r}$ times so that the expected reward of each action can be estimated within $\pm \Delta_{r}$. We will show in Lemma 4.1 that the value of $n_{r}$ is chosen to obtain high probability confidence intervals of radius $\Delta_{r}$ using Hoeffding's Inequality. At the end of each round, the estimates are used to sort the arms, where the arms $G(i), G(j)$ are considered sorted when the upper bound on reward estimate of action $\mathbf{a}_{-i}^{G}$ is less than lower bound of action $\mathbf{a}_{-j}^{G}$. When an arm is placed at its true sorted location in the group, its corresponding action is not sampled again. The procedure ends when $\Delta_{r}<\lambda$ or when all $K+1$ arms are sorted. At the end of the algorithm, only top $K$ arms are provided as output.

\subsection{MERGE}

The MERGE subroutine is given in Algorithm 3. The MERGE subroutine aims to merge two groups, each with $K$ sorted arms to give sorted best $K$ arms. Since we only want the best $K$ arms from the merged $2 K$ arms to be sorted, it can be done with only $K+1$ arm comparisons.

Starting with two $K$-sized sorted groups $G_{1}$, and $G_{2}$ and an optimal group which is empty at the start of the subroutine, we identify the best $K$ out of $2 \times K$ arms by figuring the best arm one by one. Starting with both $i$ and $j$ as 1, we construct a new action by replacing the $i^{t h}$ arm of group $G_{1}$ by the $j^{t h}$ arm of group $G_{2}$. Note that if after replacement the reward is bigger, it implies that the added $j^{t h}$ arm of $G_{2}$ is the next arm in the sorted final list, else the $i^{t h}$ arm of $G_{1}$ is the next arm in the sorted final list. In order to differentiate between the two actions, the procedure similar to the SORT subroutine is used. We sample the actions for $n_{r}$ time steps, where we will show in Lemma 4.2 that the value of $n_{r}$ is chosen to obtain high probability confidence intervals of radius

$\overline{{ }^{3} \text { We treat } \frac{N}{K+1}}$ as an integer. However, replacing $\frac{N}{K+1}$ to $\left\lceil\frac{N}{K+1}\right\rceil$ would not change the order analysis. 
$\Delta_{r}$ using Hoeffding's Inequality. Based on whether the $i^{t h}$ arm of $G_{1}$ or $j^{t h}$ arm of $G_{2}$ made in the optimal set, $i$ or $j$ is incremented and the procedure is repeated till the $K$ best arms in the merger of the two groups are obtained.

\subsection{Complexity of CMAB-SM}

We now analyze the complexity of CMAB-SM for both storage and computation at each time step. Detailed subroutines are provided in Appendix G, with the key pseudo-codes in Algorithm 1-3.

The algorithm, while running SORT or MERGE subroutine, stores the reward of each action in the group, and sorts all the actions. The total storage at any step is no more than $O(K)$. Even when the groups are being merged, $O(K)$ temporary storage is used for the merged rewards. This merged group is then used to decide the action in the exploiting phase. Thus, the maximum storage at any time is $O(K)$ for the subroutines and $O(N)$ for CMAB-SM. To evaluate the computational complexity at each time-step, we consider the three cases of what the algorithm may be doing at a time step.
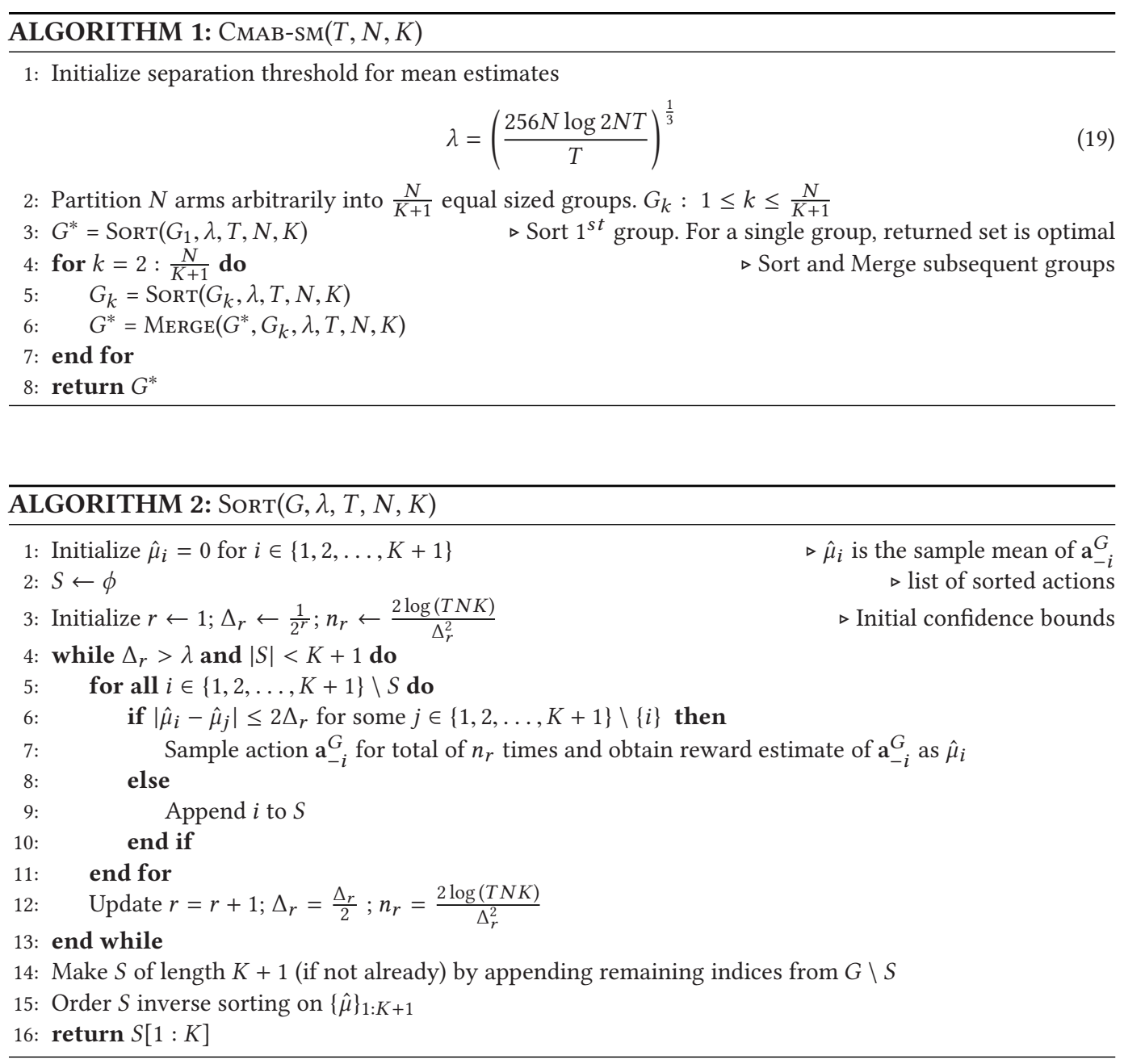


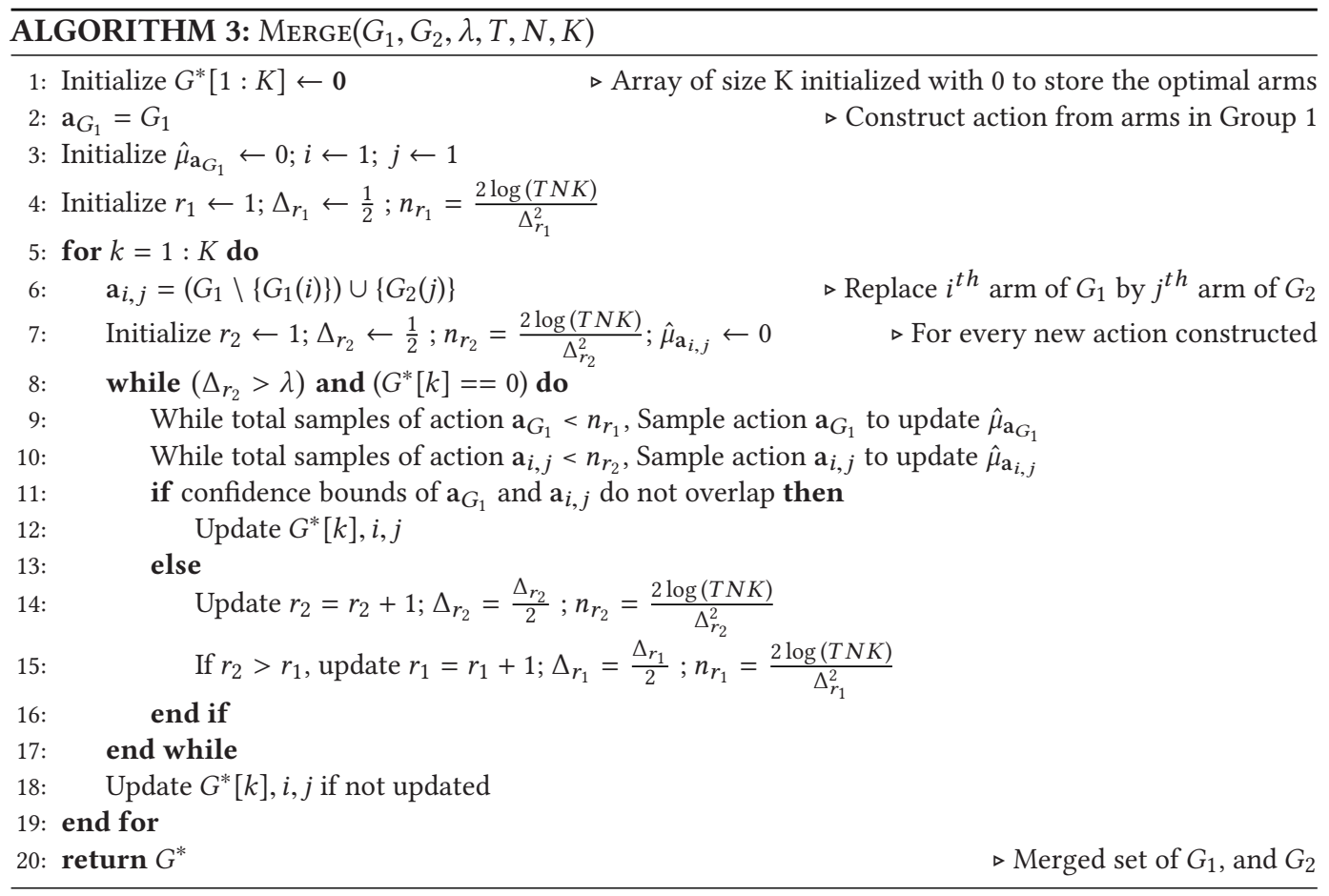

(1) In the SORT subroutine, at the end of each iteration of the while loop, arms in the group are sorted requiring $O(K \log K)$ computations. It then loops over all the arms to place them in the correct order which takes $O(K)$ steps. Thus, the computational complexity in the worst case time-step in sort is $O(K \log K)$. (2) The MERGE subroutine at any time step either runs action and saves the result, or performs comparisons which are all $O(1)$ at each time. (3) After the MERGE is complete, the best action is available which is then exploited thus making the complexity in the exploit phase as $O(1)$. Thus, the overall complexity at any time is $O(K \log K)$ which happens due to sorting the actions for the removal of sub-optimal arms after every round in the SORT subroutine.

In each call to SORT, the actions are sorted with respect to their mean observed rewards. From Lemma 3.1, an ordering is also obtained for the corresponding arms. In the MERGE subroutine, a new action is constructed from an old action by replacing exactly one arm. The ordering between the old action and the new action gives the ordering between the replaced arm and the new arm. Note that the inequality conditions work in different directions for SORT and MERGE algorithms.

\subsection{Other Design Options}

We note that an algorithm can be constructed by keeping the first $K-1$ arms fixed. The algorithm will now select the best arm from remaining $N-K+1$ arms. This arm will now always be kept in first $K-1$ arms. The process is repeated until all $K$ places are filled. However, this algorithm has two issues, (1) Higher complexity: The algorithm will need to sort $N-K+1$ arms into place which increases the sorting complexity from $\tilde{O}(K)$ to $\tilde{O}(N)$. (2) More exploration steps, since this algorithm will now perform exploration after placing every arm among the first $K$ Group. This increases the time required for exploration by a factor of $K$, which would increase the order of regret bound. This makes the CMAB-SM a better choice compared to a naïve implementation of UCB by fixing $K-1$ arms. 


$$
\delta_{G(i)}=\left\{\begin{array}{cr}
\mathbb{E}\left[X_{G(i)}\right]-\mathbb{E}\left[X_{G(i+1)}\right], & i=1 \\
\min \left\{\mathbb{E}\left[X_{G(i-1)}\right]-\mathbb{E}\left[X_{G(i)}\right], \mathbb{E}\left[X_{G(i)}\right]-\mathbb{E}\left[X_{G(i+1)}\right]\right\}, & i \in 2, \ldots, K \\
\mathbb{E}\left[X_{G(i-1)}\right]-\mathbb{E}\left[X_{G(i)}\right], & i=K+1
\end{array}\right.
$$

\section{REGRET ANALYSIS OF THE PROPOSED ALGORITHM}

In this section, we will present the main result of the paper, related to the regret analysis of the proposed algorithm.

\subsection{Bounds on Exploitation Regret and Exploration Time}

In this subsection, we will bound the regret in the exploitation phase, which indicates the loss in reward due to choosing an incorrect action at the end of the MERGE algorithm. We will also bound the time spent in the SORT and the MERGE subroutines, which is the exploration phase.

We first find the time taken in the sort and merge subroutines.

LEMma 4.1 (SORT TIME ReQUiREMENT). SORT subroutine 2 gives correct ordering on $K+1$ actions in a group $G$ with probability $1-\frac{K}{2 N^{2} T^{2}}$, up to precision $\lambda$ defined in equation (19), where the actions are chosen for at most

time steps, where $\delta_{G(i)}$ is given in (20).

$$
\left(\sum_{i=1}^{K+1} \frac{64 U^{2} \log 2 N T}{\max \left(\delta_{G(i)}^{2}, \lambda^{2}\right)}\right)
$$

Proof. (Outline) We sample each action till the confidence intervals around estimated means of any two actions are separated. The confidence intervals reduce as number of samples increases from concentration bounds from Lemma A.1 with a lower limit of $\lambda$. Using Corollary 2.7 and Hoeffding's Inequality (Lemma A.1) we bound the number of samples required for separation with high confidence. We then use union bounds to bound the total numbers of samples required for each action. The detailed proof is provided in Appendix C.

Lemma 4.2 (Merge Time Reouirement). MERGE subroutine 5 merges arms in two groups $G_{1}$ and $G_{2}$ to $G$ correctly with probability $1-\frac{K}{2 N^{2} T^{2}}$, up to precision $\lambda$ as defined in equation (19), where the total number of time steps needed to merge is at most

$$
\left(\sum_{i=1}^{K+1} \frac{64 U^{2} \log 2 N T}{\max \left(\delta_{G(i)}^{2}, \lambda^{2}\right)}\right),
$$

where $\delta_{G(i)}$ is given in (20).

Proof. (Outline) While merging two groups $G_{1}$ and $G_{2}$, we sample two actions till the confidence intervals around the estimated means of both actions are separated by twice the confidence intervals around the estimates. Again using Lemma A.1 and Corollary 2.7, we bound the number of samples required reduce the confidence intervals sufficiently enough to order two arms. The detailed proof is provided in Appendix C.

In order to bound the regret in the exploitation phase, we first characterize the probability that the action decided by CMAB-SM is not the best action.

Lemma 4.3 (Total Error Probability). The probability that the action selected by CMAB-SM during the exploitation phase is not the best action (up to precision defined in Equation 19) is at most $\frac{1}{N T^{2}}$. 
Proof. (Outline) We use union bounds to calculate total error probability of the algorithm. We use Lemmas 4.1 and 4.2 to calculate failure probability of each sort and merge event respectively. There are total $\frac{N}{K+1}$ groups to be sorted, and $\frac{N}{K+1}-1$ groups to be merged. Taking union bound over the total number of failure events, and probability of each failure event gives an upper bound on total error probability of the algorithm. The detailed proof is provided in Appendix D.

In the next result, we bound the time spent in exploring, including the SORT and MERGE subroutines for all groups.

Lemma 4.4 (Bound on Exploration Time Steps). Total time-steps used to SORT all $\frac{N}{K+1}$ groups, and merge these sorted groups one after the other is bounded as

$$
T_{\exp } \leq \frac{128 N U^{2} \log 2 N T}{\lambda^{2}}
$$

Proof. (Outline) Lemma 4.1 gives the maximum number of samples required to Sort one group. Similarly, Lemma 4.2 gives the number of samples required to merge two groups. Since there are $\frac{N}{K+1}$ groups to be sorted, and $\frac{N}{K+1}-1$ groups to be merged. Summing over total number of samples for each groups gives an upper bound on total samples required for exploration. The detailed proof is provided in Appendix E

In the following result, we bound the expected regret in the exploitation phase, caused by CMABSM selecting incorrect action.

LEMma 4.5 (Bounded ExploitATION REgRet). The expected regret when a sub-optimal action $\hat{a}^{*}$ is returned by CMAB-SM is bounded as

$$
\mathbb{E}\left[\Delta_{\hat{\mathbf{a}}^{*}}\right] \leq U \lambda \sqrt{K}+\frac{U \sqrt{K}}{N^{2} T^{2}}
$$

Proof. (Outline) Regret can arise in Exploitation phase when either SORT algorithm or MERGE algorithm had a failure event. Regret can also come in exploitation phase if two arms are have expected rewards close enough that SORT or MERGE algorithm cannot distinguish between them with high confidence. Combining these two sources of regret and using Assumption 4 gives the upper bound on the expected regret during the exploitation phase. The detailed proof is provided in Appendix F.

\subsection{Main Result}

Our main result is presented in Theorem 4.6, which states that CMAB-SM algorithm achieves a sub linear expected regret.

TheOREM 4.6. CMAB-SM algorithm described in Algorithm 1 has an expected regret accumulated during the entire time horizon upper bounded as

$$
W(T)=\tilde{O}\left(N^{\frac{1}{3}} K^{\frac{1}{2}} T^{\frac{2}{3}}\right)
$$

Proof. (Outline) We first note that regret of the algorithm for playing sub-optimal action can come during the exploration phase, or during exploitation phase if the exploration resulted in a suboptimal action. Time steps CMAB-SM uses for exploration is the total time spend in SORT and MERGE subroutines. We bound the time steps in both subroutines by $\frac{128 N U^{2} \log 2 N T}{\lambda^{2}}$ using Lemma 4.4. Further, if the algorithm results in an sub-optimal arm, the expected regret in a single time step of exploitation phase is $\left(U \lambda \sqrt{K}+\frac{U \sqrt{K}}{N T^{2}}\right)$ by using Lemma 4.5. By choosing an optimal value of $\lambda$ as defined in (19) we obtain the required bound. Having described the outline, we next give the detailed steps of the proof. 
(Detailed Steps) We note that the expected regret till time $T$ is sum of expected regret accumulated at each round. We can rewrite it as sum of two phases of the algorithm which are exploration and exploitation as,

$$
\begin{aligned}
W(T) & =\sum_{t=1}^{T} \mathbb{E}[R(t)] \\
& =\sum_{t=1}^{T_{\text {exp }}} \mathbb{E}[R(t)]+\left(T-T_{\exp }\right) \times \mathbb{E}\left[\Delta_{\hat{\mathbf{a}}^{*}}\right] \\
& \leq \sum_{t=1}^{T_{\text {exp }}} \mathbb{E}[R(t)]+T \mathbb{E}\left[\Delta_{\hat{\mathrm{a}}^{*}}\right] \\
& \leq \sum_{t=1}^{T_{\text {exp }}} \max (R(t))+T \mathbb{E}\left[\Delta_{\hat{\mathrm{a}}^{*}}\right] \\
& \leq T_{\exp } \max (R(t))+T \mathbb{E}\left[\Delta_{\hat{\mathrm{a}}^{*}}\right],
\end{aligned}
$$

where (25) follows from splitting the regret into exploration-exploitation phase, (26) follows since $T-T_{\text {exp }} \leq T$, and (27) follows since mean is at most the maximum.

Using the values for maximum regret in any round, Lemma 4.5 , inequality (70), maximum exploration time from Lemma 4.4, and maximum exploitation regret from Lemma 4.5, we have,

$$
\begin{aligned}
& W(T) \\
\leq & U \sqrt{K} \frac{128 N U^{2} \log 2 N T}{\lambda^{2}}+T\left(U \lambda \sqrt{K}+\frac{U \sqrt{K}}{N T^{2}}\right) \\
= & \left(\frac{128 N U^{3} \sqrt{K} \log 2 N T}{\lambda^{2}}+T U \lambda \sqrt{K}\right)+\frac{U \sqrt{K}}{N T} .
\end{aligned}
$$

We now choose a value of $\lambda$ to optimize $W(T)$. Since during the implementation of algorithm $U$ is most likely an unknown quantity, we use the following value of $\lambda$

$$
\lambda=\left(\frac{256 N \log 2 N T}{T}\right)^{\frac{1}{3}}
$$

Choosing the value of $\lambda$ as defined in (30), we have the total regret of the algorithm as

$$
W(T) \leq\left(U^{3}+2 U\right) \sqrt{K}(32 N \log 2 N T)^{\frac{1}{3}} T^{\frac{2}{3}}+\frac{U \sqrt{K}}{N T}
$$

This proves the result as in the statement of the Theorem.

This trick where we tune $\lambda$ after we define the precision in each SORT/MERGE round allows us to eliminate the dependence on potentially hard to order sequences of items. The intuition behind this is, in a finite time horizon, any agent wants to work out the best possible arm it can get, however can do so only up to a certain precision permitted by finite the time available.

\subsection{Handling Insufficient Exploration Time}

We note that there can be an instance where the algorithm is run with insufficient time for exploration. We first characterize what value of $T$ would result in the algorithm to run with insufficient exploration time. Then, we evaluate the regret in such a scenario. 
Note that we assumed that rewards of each arm lies between $[0,1]$ in Section 2 . This results in the gap between any two arms is less than 1. For the optimal $\lambda$ defined in Equation (19), any $T \leq 256 N \log (2 N T)$ will make $\lambda \geq 1$ which serves no practical purpose based on our assumption. In that case, we arbitrarily select one of the $\left(\begin{array}{l}N \\ K\end{array}\right)$ actions and still suffer a maximum regret of $T U \sqrt{K} \leq \lambda T U \sqrt{K}$. We have,

$$
\begin{aligned}
W(T) & \leq U \sqrt{K} T \\
& \leq T U \sqrt{K} \lambda \\
& =U \sqrt{K}(256 N \log (2 N T))^{1 / 3} T^{2 / 3}
\end{aligned}
$$

We note that the sub-linear regret in Equation (33) grow as $\tilde{O}\left(T^{2 / 3}\right)$ but with a large multiplication constant. Hence, the linear regret in Equation (31) provides a better bound because of limited time to explore all arms.

\subsection{Handling Unknown Time Horizon Using Doubling Trick}

We now analyze the case where the time horizon $T$ is unknown and the algorithm requires to optimize actions without the knowledge of $T$ to tune $\lambda$. We use the standard doubling trick from Multi-Armed Bandit literature [6, 8]. To use doubling trick we start the algorithm from $T_{0}=0$. We then restart the algorithm after every $T_{l}=2^{l}, l=1,2, \ldots$ time steps, till the algorithm reaches the unknown $T$. Each restart of the algorithm runs for $T_{l}-T_{l-1}$ steps with $T_{0}=0$ with $\lambda_{l}=$ $\left(\frac{256 N \log 2 N\left(T_{l}-T_{l-1}\right)}{T_{l}-T_{l-1}}\right)^{1 / 3}$

To show that the regret is bounded by $T^{2 / 3}$ for the doubling algorithm, we use Theorem 4 from [8] which we state in the following lemma.

Lemma 4.7. If an algorithm $\mathcal{A}$ satisfies $R_{T}\left(\mathcal{A}_{T}\right) \leq c T^{\gamma}(\log T)^{\delta}+f(T)$, for $0<\gamma<1, \delta \geq 0$ and for $c>0$, and an increasing function $f(t)=o\left(t^{\gamma}(\log t)^{\delta}(\right.$ at $t \rightarrow \infty)$, then anytime version $\mathcal{A}^{\prime}:=$ $\mathcal{D T}\left(\mathcal{A},\left(T_{i}\right)_{i \in \mathbb{N}}\right)$ with geometric sequence $\left(T_{i}\right)_{i \in \mathbb{N}}$ of parameters $T_{0} \in \mathbb{N}^{*}, b>1$, (i.e., $\left.T_{i}=\left\lfloor T_{0} b^{i}\right\rfloor\right)$ with the condition $T_{0}(b-1)>1$ if $\delta>0$ satisfies,

$$
R_{T}\left(\mathcal{A}^{\prime}\right) \leq l\left(\gamma, \delta, T_{0}, b\right) c T^{\gamma}(\log T)^{\delta}+g(T),
$$

with an increasing function $g(t)=o\left(t^{\gamma}(\log t)^{\delta}\right)$ and a constant loss $l\left(\gamma, \delta, T_{0}, b\right)>1$,

$$
l\left(\gamma, \delta, T_{0}, b\right):=\left(\left(\frac{\log \left(T_{0}(b-1)+1\right)}{\log \left(T_{0}(b-1)\right)}\right)^{\delta}\right) \times \frac{b^{\gamma}(b-1)^{\gamma}}{b^{\gamma}-1}
$$

Using Lemma 4.7 for $b=2, \gamma=2 / 3, \delta=1 / 3$, we can convert our algorithm to an anytime algorithm.

\subsection{Comparison between CMAB-SM and UCB Algorithm}

We now compare the regret bound with the one that would be achieved by using the UCB approach on each of the $\left(\begin{array}{l}N \\ K\end{array}\right)$ actions.

Lemma 4.8 (UCB Regret, [6]). For a Multi Armed Bandit setting with action space $\mathcal{A}$, time horizon T, and precision $\lambda \approx \sqrt{\frac{|\mathcal{A}| \log |\mathcal{A}|}{T}}$, the expected regret accumulated during entire time horizon $T$ using improved UCB algorithm is upper bounded as,

$$
W(T) \leq \sqrt{|\mathcal{A}| T} \frac{\log (|\mathcal{A}| \log |\mathcal{A}|)}{\sqrt{\log |\mathcal{A}|}}
$$


Bounding the size of action space by using Stirling's approximation [38], we get expected regret accumulated regret at time $T$ of UCB algorithm as,

$$
W_{U C B}(T)=\tilde{O}\left(\left(\frac{e N}{K}\right)^{\frac{K}{2}} T^{\frac{1}{2}}\right)
$$

For UCB approach to outperform CMAB-SM, $T$ has to be very large. More formally,

$$
W(T)>W_{U C B}(T), \text { when } T=\tilde{\Omega}\left(\frac{e^{3 K} N^{3 K-2}}{K^{3 K+3}}\right)
$$

Even for an agent which can play $10^{12}$ actions per second, this will take about 10 million years to outperform CMAB-SM for a setup with $N=30$ and $K=15$. Hence, for all practical problems when an agent has a large number of arms to play simultaneously, the CMAB-SM algorithm will outperform the UCB algorithm.

\subsection{Discussion of $\tilde{O}\left(T^{2 / 3}\right)$ Regret Bound}

Lower bound of $\Omega(\sqrt{N T})$ for Linear Bandits was proven in [12], and lower bound of $\Omega(\sqrt{K N T})$ is proven for semi-bandits by [28]. Note that any $\tilde{O}(\sqrt{T})$ regret bound algorithm compares all the actions with best possible action either by getting individual regret for semi-bandits or by estimating individual regret as in linear bandits $[1,12,28]$.

Individual sub-optimal action a is eliminated in $O\left(\frac{1}{\Delta_{a}^{2}}\right)$ number of samples. The regret from this action then becomes $\Delta_{\mathrm{a}} \times \frac{1}{\Delta_{\mathrm{a}}^{2}}=\frac{1}{\Delta_{\mathrm{a}}}$ and hence, the cumulative regret is of the form of $\frac{1}{\lambda} T+\lambda T$. This gives $O(\sqrt{T})$ regret for $\lambda=\frac{1}{\sqrt{T}}$.

Due to the bandit feedback, we do not directly obtain the rewards of the individual arms but we only get an ordering on any two arms which can be compared. To eliminate arms early we need some estimator of individual arms similar to linear bandits. Also, the regret accumulated to eliminate arm $i$ is not of the form $O\left(1 / \Delta_{i}\right)$. This also hinders the development of an $\tilde{O}(\sqrt{T})$ bound with linear space and time complexity.

\section{NUMERICAL EVALUATION}

In this section, we evaluate CMAB-SM under a synthetic problem setting. We compare the result with improved UCB algorithm as described in [6]. Since this paper provides the first result with non-linear reward functions for $\mathrm{CMAB}$ problem with bandit feedback, we compare with the UCB algorithm [6] which is optimal for small $N$ and $K$ while having the regret scale with $\left(\begin{array}{l}N \\ K\end{array}\right)$.

For evaluations, we ran the algorithm for $T=10^{6}$ time steps and averaged over 30 runs. We compare cumulative regret at each $t$ starting from $t=0$, which is defined as,

$$
W(t)=\sum_{t^{\prime}=1}^{t} R\left(t^{\prime}\right)
$$

We consider two values of $N \in\{12,24\}$. For $N=12$, we choose $K \in\{2,3,5\}$, while for $N=$ 24 , we choose $K \in\{2,3,5,7,11\}$. Since the arms must have FSD over each other, we describe one example single parameter distributions for the reward of each arm that have this property. We consider a random variable $Y_{i}$ which follows an exponential distribution with parameter $\lambda_{i}$, $Y_{i} \sim \exp \left(\lambda_{i}\right)$. Since this random variable can take values in $[0, \infty)$, we transform the variable using arctan function to limit it to the set $[0, \pi / 2)$ as

$$
X_{i}=\frac{2}{\pi} \arctan \left(Y_{i}\right) \text {. }
$$




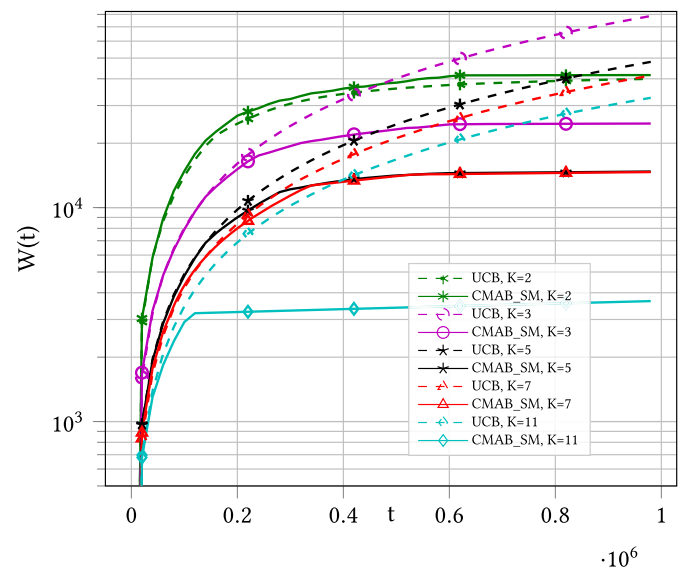

(a) $\mathrm{N}=24$

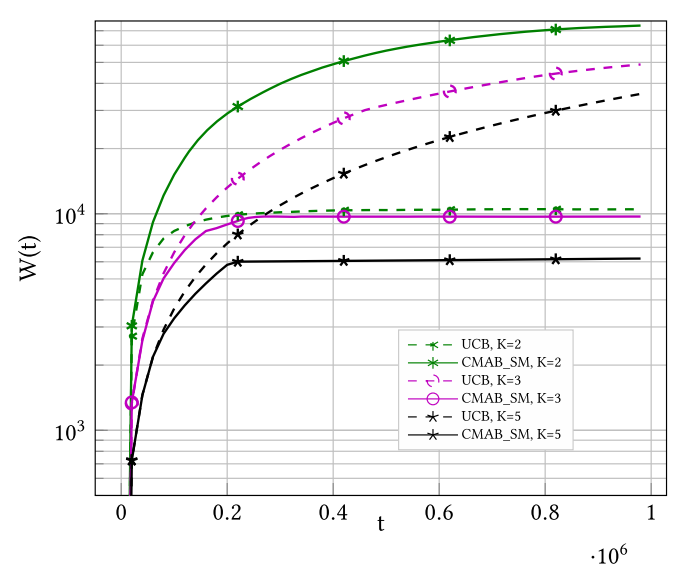

(b) $\mathrm{N}=12$

Fig. 1. Empirical regret of $U C B$ and CMAB-SM algorithm for the case when the reward of actions is a non linear function of rewards of individual arms for various values of $N$ and $K$. As it can be seen from the plots, except for the case of $K=2$, CMAB-SM incurs significantly lower regret than UCB algorithm.

We note that arm $i$ has FSD over arm $j$ if $\lambda_{i}>\lambda_{j}$. Thus, this reward distribution satisfies Assumption 2 as long as no two arms have same parameter, or $\lambda_{i} \neq \lambda_{j}$ for any $i \neq j$. Figure $3(\mathrm{~b})$ in Appendix plots $P(X \geq x)$ of the reward function for different values of $\lambda_{i}$. We see that $P(X \geq x)$ is larger for the distribution with larger value of $\lambda$, and for any two different values of $\lambda$, there is $x$ (e.g., any $x \in(0,1])$ such that $P(X \geq x)$ are not the same thus showing that the reward distributions satisfy Assumption 2.

We consider an online portal that can display $K$ products because of certain limitations. Assume that the reward, which indicates the profit from the sale of a product, from each arm follows the distribution as defined in (36). However, there is an additional benefit received when multiple products are sold together, e.g., reduced overhead/shipping costs. We define a non linear reward $r$ as a function $f$ of individual arms as follows:

$$
f\left(X_{1}, X_{2}, \ldots, X_{K}\right)=\frac{2}{K(K+1)} \sum_{i=1}^{K} \sum_{j \geq i}^{K} X_{i} X_{j} .
$$

The expected value of the reward in terms of expected value of rewards of individual arms is

$$
\mathbb{E}\left[f\left(X_{1}, X_{2}, \ldots, X_{K}\right)\right]=\frac{2}{K(K+1)}\left(\sum_{i=1}^{K} \mathbb{E}\left[X_{i}^{2}\right]+\sum_{i=1}^{K} \sum_{j>i}^{K} \mathbb{E}\left[X_{i}\right] \mathbb{E}\left[X_{j}\right]\right),
$$

This result follows from linearity of expectation. We note that the expected reward is strictly increasing with respect to the expected values of individual rewards. Figure 1 shows the evaluation results for setting where the reward of an action is the function described in Equation (37) of the rewards of individual rewards of the arms. We see that for both values of $N$, CMAB-SM outperforms UCB for $K>3$ in the time step range considered. Further, for $N=24$ and $K=2$, the gap between the proposed algorithm and UCB is small. In summary, when the value of $\left(\begin{array}{l}N \\ K\end{array}\right)$ is moderately large, and $T$ is not significantly large $\left(T<\tilde{O}\left(\frac{e^{3 K} N^{3 K-2}}{K^{3 K+3}}\right)\right)$, CMAB-SM outperforms the baseline. Further, the computation and storage complexity of the proposed algorithm are much better as compared to the baseline, as seen in Section 3.3. 
In Appendix H, we further consider two more examples for Bernoulli distribution, where the sum and maximum of rewards are considered as the reward functions.

\section{APPLICATION TO SOCIAL INFLUENCE MAXIMIZATION}

We now apply CMAB-SM and evaluate it for solving the problem of Social Influence Maximization, which in the words of Domingos \& Richardson [15] is defined as follows: "if we can try to convince a subset of individuals in a social network to adopt a new product or innovation, and the goal is to trigger a large cascade of further adoptions, which set of individuals should we target?"

\subsection{Problem Description}

Consider a social network $G=(V, E, P)$, where $V$ is the node set representing individuals, $E=$ $\{(u, v): u, v \in V\}$ is the set of directed ( $u$ to $v$ ) connections among the individuals, and $P=\left\{p_{e}\right.$ : $e \in E\}$ is the set the strengths/weights of the connections in $E$.

For the standard influence maximization problem [23], we select a 'seed' set $S$ of $K>0$ individuals to initiate a cascade. Analogous to diseases spreading between individuals, the set of users $S$ will attempt to influence their neighbors in the social network $G$, and those infected neighbors will attempt to influence their neighbors, and so on. We consider the simple, discrete-time independent cascades diffusion model. In this model, if individual $u$ becomes influenced, then at the next time step, individual $u$ attempts to influence each of his neighbors $v$ in the network with probability $p_{(u, v)}$. All the influence attempts are statistically independent of each other. Our goal in picking the seed set $S$ is to have a large cascade. Let $y^{(v)}=1$ denote that node $v$ was influenced in the cascade and $y^{(v)}=0$ otherwise. Let $\sigma(S)$ denote the expected cascade size when $S$ is the seed set,

$$
\sigma(S)=\mathbb{E}\left[\sum_{v \in V} y^{(v)} \mid \text { individuals in } S \text { were initially influenced }\right] \text {. }
$$

The goal is to find $\arg \max _{S \subseteq V:|S|=K} \sigma(S)$.

Note that the word time-step in the above diffusion model is different from the word time-step in an adaptive/online algorithm. In fact, for every time-step in an adaptive algorithm, the entire diffusion (all time-steps of the aforementioned independent cascade model) takes place.

In the traditional problem, $G$ is fully known, not only the edge set $E$ but also the diffusion parameters $P$. Here, we consider an adaptive version of the problem, where only the individuals (node set $V$ ) are known. At each time $t$, we select a seed set $S_{t}$ and observe a single resulting cascade $\left\{y_{t}^{(v)}\right\}_{v \in V}$. Using the information from those outcomes, at time $t+1$ we select a (possibly new) seed set, and so on. We want to maximize the average, expected

$$
\underset{\left\{S_{t} \subseteq V:\left|S_{t}\right|=K\right\}_{t=1}^{T}}{\arg \max } \frac{1}{T} \quad \sum_{t=1}^{T} \mathbb{E}\left[\sum_{v \in V} y_{t}^{(v)} \mid \text { individuals in } S_{t} \text { were initially influenced }\right]
$$

Recent works on adaptive influence maximization assume semi-bandit feedback, such as which specific nodes were influenced or even along which edges influence propagated [39, 41]. In [30], $\epsilon$-greedy strategies are proposed for adaptive influence maximization although assumptions are made on edge parameter distributions.

\subsection{Algorithms}

We propose to solve the adaptive influence maximization problem using CMAB-SM. Note that $\mathrm{CMAB}-\mathrm{SM}$ is a general procedure for $\mathrm{CMAB}$ problems and does not use any special properties of network diffusion models or knowledge about the edge set $E$ or parameters $P$. 
The reward $r_{\mathrm{a}}(t)$ for an action a of choosing seed set $S$ at time $t$ is the count of infected individuals in a cascade run at time $t$ where only nodes in $S$ were initially infected,

$$
r_{\mathbf{a}}(t)=\sum_{v \in V} y_{t}^{(v)} \text { where only } v \in S \text { were initially influenced. }
$$

The aggregate reward function $r_{\mathrm{a}}(t)$ cannot be decomposed into a function of "rewards" for individual nodes like $f\left(\mathbf{d}_{\mathbf{a}_{t}}\right)$ in (1). Thus, it does not strictly satisfy the assumptions used to prove performance guarantees for CMAB-SM. However, the reward function $r_{\mathrm{a}}(t)$ satisfies properties related to those in the assumptions. We discuss this in more detail in Appendix I.

We compare the performance of CMAB-SM against an $\epsilon$-greedy version of the credit distribution model [20], we call it $\epsilon$-CD. The credit distribution model was proposed to infer influences from historical data without knowledge of the diffusion parameters. It does require the edge set $E$; to provide a fair comparison to CMAB-SM, we provide the credit distribution method with the edge set of the complete directed graph (for which edges $e^{\prime} \notin E$ have $p_{e^{\prime}}=0$ ).

The $\epsilon$-CD algorithm follows standard $\epsilon$-greedy method for bandits. For a fraction $\epsilon$ of the total time horizon $T$, a random size $K$ subset of nodes is chosen. For the rest of the time horizon it exploits the best seed set of size $K$ chosen by the credit-distribution algorithm using the diffusion history obtained from $\epsilon T$ time steps. The credit-distribution algorithm uses the list of infected nodes from the $t-1$ previous cascades and when exactly did they get infected within those cascades to estimate the expected reward of different size $K$ seed sets. The seed set it estimates as best is then used at time $t$. Note that the proposed algorithm $\mathrm{CMAB}$ uses more limited feedback-only the number of nodes infected in each cascade.

It is also of interest to understand how close does CMAB-SM get to the actual optimal set. However, for large networks, it can be computationally intractable to find the optimal set. Hence, instead of the optimal set, we compare CMAB-SM with the greedy-best optimal set obtained by the simulation-based greedy algorithm which is guaranteed [23] to have an influence of at least $1-\frac{1}{e}$ times the influence of the actual optimal set. Moreover, the simulation-based greedy algorithm is computationally very expensive as compared to CMAB-SM as it involves large number of costly Monte-Carlo simulations.

Note that, contrary to the cross-selling application discussed in Section 5, we do not use the LinUCB algorithm for our comparisons as it is not scalable for large values of $N$.

\subsection{Experimental Setup}

For our experiments, we considered the Facebook Friends network [31] which has 4,039 nodes with 88,234 undirected edges. We used a representative community (sub-network) of the Facebook network which has 534 nodes and 8,158 undirected edges. It was identified using the the Louvain method for community detection [9]. The selected community originally had one node with extremely high degree of 533 within the selected community, which was removed. Each undirected edge was then replaced by two directed edges. Following [23], for each node $v \in V$, the parameter of each edge entering $v$ was set to $1 /$ indegree $(v)$. We used a time horizon of $T=100,000$. At each time step $t$, each method selected a seed set $S_{t}$, which was used to run a single cascade. The total number of influenced users was set as the reward.

For the $\epsilon$-CD algorithm, we used the exploration probabilities $\epsilon=0.10,0.25$. For the offline greedy algorithm, we averaged 1,000 Monte-Carlo diffusions for each candidate subset.

We used seed-set sizes $K=2,4,8$. Performance of each algorithm was measured using observed reward, averaged over 10 runs. For aesthetic reasons, we smoothed the average across runs using a simple moving average of the data for the previous 500 time points. 


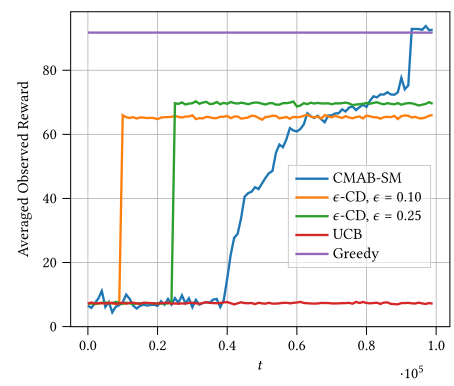

(a) $K=2$

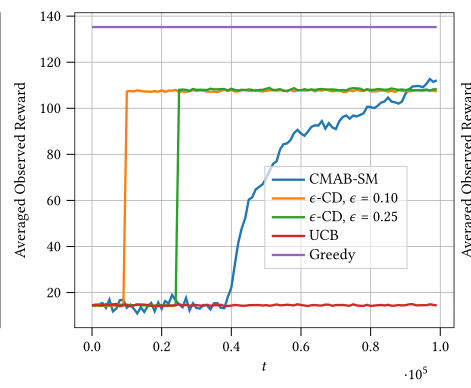

(b) $K=4$

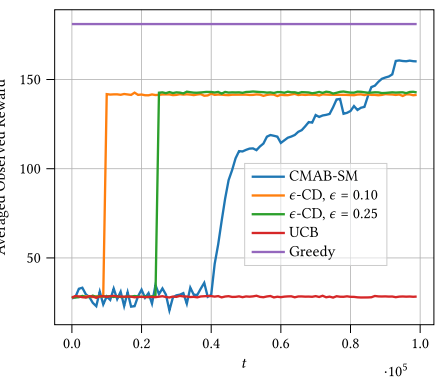

(c) $K=8$

Fig. 2. Averaged observed reward for different algorithms for different seed set sizes for the Facebook friends network.

For the simulation-based greedy algorithm, we have calculated the expected influence of any subset as Monte-Carlo average of the observed influences based on 1,000 diffusions.

\subsection{Results}

The observed reward for each algorithm, averaged over 10 independent runs and further smoothed using moving average filter on the time-axis, is shown in Figure 2, separately for each $K=2,4,8$.

For all values of $K$, CMAB-SM significantly out-performs the UCB algorithm. For the size of this problem $(N=534$ and $K \in\{2,4,8\})$, the UCB algorithm is unable to even finish sampling each possible set of $K$ nodes once, even for $K=2$. Thus, although for sufficiently large $T$ the UCB algorithm should eventually find the optimal set, it needs a much larger time horizon $T$ to do so. The efficient divide-and-conquer strategy of CMAB-SM allows it to do well while the exhaustivesearch based UCB algorithm is still in the exploration phase.

For all values of $K, \mathrm{CMAB}-\mathrm{SM}$ achieves a lower cumulative reward than the $\epsilon$-CD algorithms (area under the curves in Figure 2). Recall that the $\epsilon$-CD algorithms are domain-specific and receive significantly more feedback than our proposed domain-agnostic algorithm CMAB-SM does, so we expect that the $\epsilon$-CD algorithms would do better. We note that the CMAB-SM algorithm is in SORT phase until around $t=4 \times 10^{4}$. The CMAB-SM algorithm finishes exploration around $t=9 \times 10^{4}$ which is consistent with Lemma 14 of the main text with $\lambda$ defined in Equation (19).

However, despite CMAB-SM being domain-agnostic and receiving less feedback, the final seed sets selected by CMAB-SM are significantly better than those selected by the $\epsilon$-CD algorithms for $K=2$ and $K=8$, and slightly better for $K=4$. In fact, for $K=2$, despite having no explicit knowledge of the underlying network or diffusion dynamics, and receiving minimal feedback, CMAB-SM finds the same set as the greedy offline method, which is provably near-optimal. This result may in part be due to probabilistic stochastic dominance holding with a high probability, especially for $K=2$.

We do not formally compare empirical run-times of the different algorithms because we wrote Python3 implementations for CMAB-SM and UCB, but for $\epsilon$-CD, we used Python3 code that called an executable for the credit distribution method [19] which required extensive disk I/O. In Table 1, we provide complexity bounds, suggesting $\epsilon$-CD is inherently slower than CMAB-SM. In terms of both time and space complexity, CMAB-SM algorithm outperforms $\epsilon$-CD algorithm (a domainspecific algorithm) and UCB algorithm (a bandit-specific algorithm).

We compare the amortized time-complexity for all the algorithms. We do so because exactly once for the entire time-horizon, the $\epsilon$-CD algorithm scrapes through the history of independentcascade diffusions accumulated over $\epsilon T$ time steps where each diffusion can be of the length $N$ in the worst-case. Hence, $\epsilon N T$ amount of time consumed by this bottleneck step is effectively 
Table 1. Computational Complexities of the Different Algorithms

\begin{tabular}{ccc}
\hline \hline Algorithm & Amortized Time-Complexity & Worst-Case Space-Complexity \\
\hline CMAB-SM & $O(K \log K)$ & $O(N)$ \\
$\epsilon$-CD & $\Omega(\epsilon N)$ & $\Omega(\epsilon N T)$ \\
UCB & $O\left(N^{K} K \log N\right)$ & $O\left(N^{K}\right)$ \\
\hline
\end{tabular}

distributed over $T$ time steps. However, the space-complexity cannot be distributed across all time steps, as we would need a computer of at least worst-case space-complexity to store the history of independent-cascade diffusions accumulated over $\epsilon T$ time steps. Hence, we compare the worstcase space complexity for all algorithms.

Remark 3. For sufficient exploration time, there is an additional factor of $T^{-\frac{1}{3}}$ in amortized timecomplexity of the CMAB-SM algorithm as the exploration, and sorting of actions, only happens for $O\left(T^{\frac{2}{3}}\right)$ steps.

\section{CONCLUSIONS AND FUTURE WORK}

This paper considers the problem of combinatorial multi-armed bandits with non-linear rewards, where agent chooses $K$ out of $N$ arms in each time-step and receives an aggregate reward. A novel algorithm, called CMAB-SM, is proposed which is shown to be computationally efficient and has a space complexity which is linear in number of base arms. The algorithm is analyzed in terms of a regret bound, and is shown to outperform the approach of considering the combinatorial action as the arm for limited time horizon T. CMAB-SM provides a way to resolve two challenges in the combinatorial bandits problem. The first is that the feedback is non-linear in the individual arms, and the second is that the space complexity in the previous approaches could be large due to exploding action space.

CMAB-SM works efficiently for large $N$ and $K$. However, finding an algorithm which is efficient in space complexity, having a regret bound which increases as $T^{1 / 2}$ instead of $T^{2 / 3}$, and not having combinatorial factors in the regret bound, is an important research direction. Followed by our work, this problem was considered in [3], where an accept-reject based algorithm is proposed that achieves a regret of $\widetilde{O}(K \sqrt{K N T})$. We note that even though the bound on $T$ is better in [3], the bounds on $K$ and $N$ are not. Indeed, our bounds are better when $T<\widetilde{O}\left(N K^{6}\right)$. Due to such high requirement of $T$ for [3], we note that their approach does not outperform the approach in this paper for the social influence maximization. Thus, an algorithm that is efficient in all parameters $N, K$, and $T$ is left for the future. Considering non-symmetric functions of individual rewards and studying the applications of more general settings to Social Influence Maximization remains as a future work.

\section{APPENDICES}

\section{A FUNDAMENTAL LEMMAS}

In this subsection, we will describe some lemmas that will be later used to prove the regret bound in Theorem 1. The first lemma is the Hoeffding's Inequality, which will be used in the results in this paper.

Lemma A.1 (Hoeffoing's Inequality [24]). Let $X$ be a random variable bounded in $[a, b]$, and let $\bar{X}$ denote the expected value of $X$. Further, let $\hat{\bar{X}}$ denote the average of $n$ i.i.d. samples of $X$. Then, for any $\epsilon>0$, we have

$$
P(|\bar{X}-\hat{\bar{X}}| \geq \epsilon) \leq 2 e^{-\frac{2 n \epsilon^{2}}{(b-a)^{2}}}
$$


The next result shows that the property of FSD is preserved by strictly increasing functions.

LEMmA A.2. Suppose that a random variable $X$ has FSD over another random variable $Y$ (or $X>Y$ ). Further, let $g^{\prime}(\cdot)$ be a strictly increasing function on $\mathbb{R}$. Then, $g^{\prime}(X)$ has FSD over $g^{\prime}(Y)$, or

$$
g^{\prime}(X)>g^{\prime}(Y)
$$

Proof. Since $X>Y$, we have

$$
P(X \geq x) \geq P(Y \geq x)
$$

Transforming $X$ and $Y$ using the function $g^{\prime}$, and using the strict monotonicity of $g^{\prime}$, we have

$$
P\left(g^{\prime}(X) \geq g^{\prime}(x)\right) \geq P\left(g^{\prime}(Y) \geq g^{\prime}(x)\right)
$$

Taking $t \triangleq g^{\prime}(x)$, we have,

$$
P\left(g^{\prime}(X) \geq t\right) \geq P\left(g^{\prime}(Y) \geq t\right)
$$

By the same arguments, if there is an $x$ where $P(X \geq x)>P(Y \geq x)$, for $t=g^{\prime}(x), P\left(g^{\prime}(X) \geq t\right)>$ $P\left(g^{\prime}(Y) \geq t\right)$. Thus, we have $g^{\prime}(X)>g^{\prime}(Y)$.

\section{B PROOF OF LEMMA 3.1}

Note we can write the actions using replacement function $h$ to replace reward of $G(j)$ arm by the reward of $G(i)$ arm, and obtain

$$
\mathbf{d}_{\mathbf{a}_{-j}^{G}}=h\left(\mathbf{d}_{\mathbf{a}_{-i}^{G}}, j, X_{G(i)}\right) \forall j
$$

The expectation of the reward of an action formed using replacement can be written as expected value of the conditional expectation of the replaced reward value of arm $G(i)$. More precisely,

$$
\mathbb{E}\left[f\left(\mathbf{d}_{\mathbf{a}_{-j}}\right)\right]=\mathbb{E}\left[\mathbb{E}\left[f\left(h\left(\mathbf{d}_{\mathbf{a}_{-i}^{G}}, j, X_{G(i)}\right)\right) \mid X_{G(i)}\right]\right]
$$

In the right hand side of Equation (42), the outer expectation is taken over $X_{G(i)}$ while the inner expectation is over $\mathbf{d}_{\mathrm{a}_{-i}^{\mathrm{G}}}$. In addition, we can replace the reward of an arm $G(j)$ in $\mathbf{d}_{\mathrm{a}_{-i}^{G}}$ by itself to obtain

$$
\mathbf{d}_{\mathrm{a}_{-i}^{G}}=h\left(\mathbf{d}_{\mathrm{a}_{-i}^{G}}, j, X_{G(j)}\right)
$$

Similar to (42), we can write the expected reward of the action as the expectation of conditional expectation of reward of arm $G(j)$. Thus, we have

$$
\mathbb{E}\left[f\left(\mathbf{d}_{\mathbf{a}_{-i}^{G}}\right)\right]=\mathbb{E}\left[\mathbb{E}\left[f\left(h\left(\mathbf{d}_{\mathbf{a}_{-i}^{G}}, j, X_{G(j)}\right)\right) \mid X_{G(j)}\right]\right]
$$

The ordering between actions $\mathbf{a}_{-i}^{G}$ and $\mathbf{a}_{-j}^{G}$ is defined as an order between the expected rewards of the respective actions. We assume that $\mathbf{a}_{-i}^{G}$ provides a higher expected reward than $\mathbf{a}_{-j}^{G}$, hence we have $\mu_{\mathbf{a}_{-i}^{G}}>\mu_{\mathrm{a}_{-j}^{G}}$. This further implies

$$
\mathbb{E}\left[f\left(\mathbf{d}_{\mathbf{a}_{-i}^{G}}\right)\right]>\mathbb{E}\left[f\left(\mathbf{d}_{\mathbf{a}_{-j}^{G}}\right)\right]
$$

Replacing the right hand side of (45) by (42) and the left hand side of (45) by (44), we have

$$
\begin{aligned}
& \mathbb{E}\left[\mathbb{E}\left[f\left(h\left(\mathbf{d}_{\mathbf{a}_{-i}^{G}}, j, X_{G(j)}\right)\right) \mid X_{G(j)}\right]\right] \\
& >\mathbb{E}\left[\mathbb{E}\left[f\left(h\left(\mathbf{d}_{\mathrm{a}_{-i}^{G}}, j, X_{G(i)}\right)\right) \mid X_{G(i)}\right]\right]
\end{aligned}
$$


We define a function $g(x)$ as the conditional expectation of reward function $f(\cdot)$ with respect to the reward of the $j^{t h}$ arm being $x$. More precisely,

$$
g(x) \triangleq \mathbb{E}\left[f\left(h\left(\mathbf{d}_{\mathbf{a}_{-i}^{G}}, j, X\right)\right) \mid X=x\right]
$$

Replacing the conditional expectations in (46), by $g$ as defined in (47), we get

$$
\mathbb{E}_{X_{G(j)}}\left[g\left(X_{G(j)}\right)\right]>\mathbb{E}_{X_{G(i)}}\left[g\left(X_{G(i)}\right)\right]
$$

From Assumption 3, $g(x)$ is a strictly increasing function of $x$, and from Assumption 2, rewards of individual arms have FSD relationships. Using Assumptions 3 and 2 along with equation (48), we want to prove that arm $G(j)$ has FSD over arm $G(i)$. Let us assume the converse where arm $G(i)$ has FSD over arm $G(j)$, or $X_{G(i)}>X_{G(j)}$. Due to the strict increasing nature of $g(\cdot)$ and Lemma A.2, we have $g\left(X_{G(i)}\right)>g\left(X_{G(j)}\right)$. Using Lemma 2.4, we further have

$$
\mathbb{E}\left[g\left(X_{G(i)}\right)\right] \geq \mathbb{E}\left[g\left(X_{G(j)}\right)\right]
$$

This inequality (49) does not agree with the inequality obtained from the original assumption (48) thus disproving $X_{G(i)}>X_{G(j)}$. Since every two arms have FSD relation and $X_{G(i)}>X_{G(j)}$ does not hold, we have

$$
X_{G(j)}>X_{G(i)}
$$

Hence, an ordering on the expected rewards of the $K+1$ actions constructed by leaving one arm aside, gives an ordering on the $K+1$ arms of the group.

\section{NUMBER OF TIME STEPS IN SORT AND MERGE SUBROUTINES}

In this subsection, we will bound the number of exploration time steps that are spent in SORT and MERGE subroutines. The next result bounds the number of time steps spent in a group $G$ in the SORT subroutine to obtain an ordering on the actions and thus on arms (by Lemma 3.1).

Proof of Lemma 4.1. Let $G(i)$ for all $i \in 1,2, \ldots, K+1$ be the different arms of group $G$ that we aim to sort using the SORT subroutine. Let $\hat{\mu}_{\mathrm{a}_{-i}^{G}}$ be the estimate of action made using all arms in $G$ except arm $G(i)$. We want to identify the number of time steps spent in SORT subroutine, and the error probability in the ordering.

The algorithm proceeds in rounds starting from $r=1$. We define $\Delta_{r} \triangleq 2^{-r}$, and each un-sorted action in round $r$ is played for $n_{r} \triangleq \frac{\log 2 N T}{\Delta_{r}^{2}}$ time steps. Using Hoeffding's Inequality, the expected reward estimate of each action lies in the range $\left[\hat{\mu}_{\mathrm{a}_{-i}^{G}}-\Delta_{r}, \hat{\mu}_{\mathrm{a}_{-i}^{G}}+\Delta_{r}\right]$ with probability bound given as

$$
\begin{aligned}
P\left(\left|\mu_{\mathrm{a}_{-i}^{G}}-\hat{\mu}_{\mathrm{a}_{-i}}\right| \geq \Delta_{r}\right) & \leq 2 e^{-2 \frac{\log 2 N T}{\Delta_{r}^{2}} \Delta_{r}^{2}} \\
& =\frac{1}{2 N^{2} T^{2}}
\end{aligned}
$$

Thus, the expected reward estimate of each action lies in the range $\left[\hat{\mu}_{\mathrm{a}_{-i}^{G}}-\Delta_{r}, \hat{\mu}_{\mathrm{a}_{-i}^{G}}+\Delta_{r}\right]$ with probability at least $1-\frac{1}{2 N^{2} T^{2}}$. Let it take $r_{i}$ rounds to be able to sort $\mathrm{a}_{-i}^{G}$ in its correct position, which implies that all other actions can be well separated from this action. When two actions are separated, the upper confidence bound for the action with a lower estimated reward is less than the lower confidence bound of action with higher estimated reward, which gives the following inequality

$$
\hat{\mu}_{\mathrm{a}_{-i}^{G}}+\Delta_{r_{i}}<\hat{\mu}_{\mathrm{a}_{-j}^{G}}-\Delta_{r_{i}} .
$$


This further means that the actions $\mathbf{a}_{-i}^{G}$ and $\mathbf{a}_{-j}^{G}$ were inseparable in round $r_{i}-1$, thus the following holds.

$$
\hat{\mu}_{\mathrm{a}_{-i}^{G}}+\Delta_{r_{i}-1} \geq \hat{\mu}_{\mathrm{a}_{-j}^{G}}-\Delta_{r_{i}-1}
$$

However, using Lemma A.1, $\hat{\mu}_{\mathrm{a}_{-i}^{G}}$ lies between the confidence region around the true mean as $\hat{\mu}_{\mathrm{a}_{-i}^{G}} \in\left[\mu_{\mathrm{a}_{-i}^{G}}-\Delta_{r_{i}-1}, \mu_{\mathrm{a}_{-i}^{G}}+\Delta_{r_{i}-1}\right]$. The same is true for $\hat{\mu}_{\mathrm{a}_{-j}^{G}}$. Using the upper limits of estimated mean rewards, we can rewrite inequality (52) as

$$
\mu_{\mathrm{a}_{-i}^{G}}+2 \Delta_{r_{i}-1} \geq \mu_{\mathrm{a}_{-j}^{G}}-2 \Delta_{r_{i}-1}
$$

From inequality (53), we get $\Delta_{r_{i}}$ in terms of the difference of expected rewards of the two arms as follows.

$$
\begin{aligned}
4 \Delta_{r_{i}-1} & \geq \mu_{\mathrm{a}_{-j}^{G}}-\mu_{\mathrm{a}_{-i}^{G}} \\
& \geq \frac{1}{U}\left(\mathbb{E}\left[X_{G(i)}\right]-\mathbb{E}\left[X_{G(j)}\right]\right),
\end{aligned}
$$

where the last step follows from Corollary 2. Using the definition of $\Delta_{r}$ and the upper bound obtained in inequality (54), we have

$$
\begin{aligned}
2^{-\left(r_{i}-1\right)} & =\Delta_{r_{i}-1} \\
& \geq \frac{\left(\mathbb{E}\left[X_{G(i)}\right]-\mathbb{E}\left[X_{G(j)}\right]\right)}{4 U}
\end{aligned}
$$

Taking logarithm with base 2, we obtain a lower bound on the number of rounds needed as

$$
r_{i}-1 \leq \log _{2}\left(\frac{4 U}{\left(\mathbb{E}\left[X_{G(i)}\right]-\mathbb{E}\left[X_{G(j)}\right]\right)}\right) .
$$

The term $\left(\mathbb{E}\left[X_{G(i)}\right]-\mathbb{E}\left[X_{G(j)}\right]\right)$ will be lowest for $j=i+1$ or $i-1$ since the arms closest to $i$ will result in the lowest gap. Thus, we define $\delta_{i}$ for arm $G(i)$ as

$$
\begin{aligned}
\delta_{i} \triangleq & \min \left\{\mathbb{E}\left[X_{G(i)}\right]-\mathbb{E}\left[X_{G(i+1)}\right],\right. \\
& \left.\mathbb{E}\left[X_{G(i-1)}\right]-\mathbb{E}\left[X_{G(i)}\right]\right\}
\end{aligned}
$$

The number of rounds to correctly rank arm $G(i)$ can thus be upper bounded as follows.

$$
r_{i} \leq \log _{2}\left(\frac{8 U}{\delta_{i}}\right) .
$$

Having bounded the number of rounds to rank $\mathbf{a}_{-i}^{G}$, we find the number of time steps each action is played till round $r_{i}$ as follows.

$$
\begin{aligned}
n_{r_{i}} & =\frac{\log 2 N T}{\Delta_{r_{i}}^{2}} \\
& =2^{2 r_{i}} \log 2 N T \\
& \leq 2^{2 \log _{2}\left(\frac{8 U}{\delta_{i}}\right)} \log 2 N T \\
& =\frac{64 U^{2} \log 2 N T}{\delta_{i}^{2}}
\end{aligned}
$$

This provides the number of times action $\mathbf{a}_{-i}^{G}$ is played. Let $\lambda$ be chosen as a threshold, which is the precision level below which we cannot correctly order two actions. Using $\lambda$ as a lower bound 
for $\delta_{i}$, each of the $K+1$ action is chosen for $n_{r_{i}}$ times, where $\delta_{i}$ is replaced by $\max \left(\delta_{i}, \lambda\right)$. Thus, the total time steps any of the action is selected is given as

$$
n^{\prime} \leq \sum_{i=1}^{K+1} \frac{64 U^{2} \log 2 N T}{\max \left(\delta_{i}^{2}, \lambda^{2}\right)}
$$

which proves the number of time steps as in the statement of the Lemma.

For $K+1$ arms, there can be $\left(\begin{array}{c}K+1 \\ 2\end{array}\right)$ ordered pairs denoting the edges of a complete graph, where each edge is the ordering of the two vertices. However, the ordering is preserved by a sub-graph which is a chain by just keeping the edges which connect two immediately ordered vertices. The number of edges thus becomes $(K+1)-1=K$. Thus, there are $K$ orderings in a group, and all of them should be correct to make the algorithm work. The probability of error in sorting group $G$ can then be written as

$$
\begin{aligned}
& P(\{\text { any arm is incorrectly sorted }\}) \\
= & 1-P(\{\text { all arms are correctly sorted }\}) \\
\leq & 1-\left(1-\frac{1}{2 N^{2} T^{2}}\right)^{K} \\
< & 1-\left(1-\frac{K}{2 N^{2} T^{2}}\right) \\
= & \frac{K}{2 N^{2} T^{2}},
\end{aligned}
$$

where the probability that any two arms are incorrectly sorted is bounded by $\frac{1}{2 N^{2} T^{2}}$ as given in (51). This proves the probability of correct ordering as in the statement of the Lemma.

Having understood the number of time steps spent in the SORT subroutine, and the error probability of ranking arms in each group, we now consider the MERGE subroutine. In the following lemma, we find the number of time steps it takes to merge two groups, with an error probability on the ordering in the combined group.

Proof of Lemma 4.2. In the MERGE subroutine, we use the sorted groups $G_{1}$ and $G_{2}$ to construct a group $G$ of $K$ elements such that arms in group $G$ are sorted. We maintain two counters $i$ and $j$ for the groups $G_{1}$ and $G_{2}$, respectively. We replace arm $G_{2}(j)$ by $G_{1}(i)$ in group $G_{1}$ to create a new action. We play both actions in rounds starting from $r=1$. At the end of round $r$, the deviation of estimated mean reward and true expected rewards is $\Delta_{r} \triangleq 2^{-r}$. By round $r$, each action has been played for $n_{r} \triangleq \frac{\log 2 N T}{\Delta_{r}^{2}}$ time steps similar to Algorithm 2. Two actions are separated when the upper confidence bound of worse action is lesser than the lower confidence bound of better action. We then add the arm corresponding to better action to group $G$ and increment the counter for the corresponding group from which arm was picked and continue comparing new actions. This is continued till we have $K$ arms in $G$.

Since the fundamental concept of comparison of two actions in Algorithm 2 is the same as that in Algorithm 3, similar analysis follows and the number of time steps required to merge the groups is

$$
n^{\prime} \leq \sum_{i=1}^{K+1} \frac{64 U^{2} \log 2 N T}{\max \left(\delta_{G(i)}^{2}, \lambda^{2}\right)},
$$

where $G(K+1)$ is the last arm with which comparison was made but not added to group $G$. The same argument as in the proof of Lemma 4.1 can be used to bound the error probability of a single run of Algorithm 3 by $\frac{K}{2 N^{2} T^{2}}$. 


\section{PROOF OF LEMMA 4.3}

To bound the probability of error of Algorithm 1, we define the event $\mathcal{E}$ which is the event when the algorithm makes an error. The algorithm makes an error when either the SORT subroutine, the MERGE subroutine, or both, make an error. So, we can write the error event as a union of error events in sorting and error events in merging. Let $\mathcal{E}_{s}$ the event where at least one of the $\frac{N}{K+1}$ calls made to Algorithm 2 resulted in an incorrect list, and $\mathcal{E}_{m}$ be the event where at least one of the $\frac{N}{K+1}-1$ merges is in error.

$$
\mathcal{E}=\mathcal{E}_{s} \cup \mathcal{E}_{m}
$$

Since the probability of the union of events is upper bounded by the sum of probabilities of individual events, we get

$$
P(\mathcal{E}) \leq P\left(\mathcal{E}_{s}\right)+P\left(\mathcal{E}_{m}\right)
$$

We now identify upper bounds for $P\left(\mathcal{E}_{s}\right)$ and $P\left(\mathcal{E}_{m}\right)$ by breaking down the error events into error in each call to the SORT and MERGE subroutines.

Let us define an event $\mathcal{E}_{s, i}$ which denotes that there was an error in the sorted list given by Algorithm 2 for $i^{\text {th }}$ group, or

$$
\begin{aligned}
\mathcal{E}_{s, i} \triangleq & \{\text { at least two arms are incorrectly placed in } \\
& \text { sorting } \left.i^{\text {th }} \text { group }\right\}
\end{aligned}
$$

Hence, $\mathcal{E}_{s}$ is a union of $\mathcal{E}_{s, i}$ for all $i \in\left\{1,2, \ldots, \frac{N}{K+1}\right\}$, or $\mathcal{E}_{s}=\bigcup_{k=1}^{\frac{N}{K+1}} \mathcal{E}_{s, i}$. Probability of error in any of the sorting operations is thus given as

$$
\begin{aligned}
P\left(\mathcal{E}_{s}\right) & =P\left(\bigcup_{k=1}^{\frac{N}{K+1}} \mathcal{E}_{s, i}\right) \\
& \leq \sum_{k=1}^{\frac{N}{K+1}} P\left(\mathcal{E}_{s, i}\right) \\
& <\sum_{k=1}^{\frac{N}{K+1}} \frac{K}{2 N T^{2}} \\
& =\frac{N}{K+1} \frac{K}{2 N^{2} T^{2}} \\
& <\frac{1}{2 N T^{2}}
\end{aligned}
$$

where (58) and (59) follow from the definition of events and the union bound, respectively, and (60) follows from Lemma 4.1.

Similarly, we define event $\mathcal{E}_{m, i}$ representing that Algorithm 2 incorrectly merges the merged group up to group $i$ and $(i+1)^{t h}$ group. Let $G_{M}^{i}$ be the merged sorted group formed by merging sorted groups $G_{M}^{i-1}$ and $G_{i}$ for $i>1$, with $G_{M}^{1}=G_{1}$. Then, we have

$$
\mathcal{E}_{m, i}=\left\{\text { error occurred while merging } G_{M}^{i} \text { and } G_{i+1}\right\} .
$$


We note that $\mathcal{E}_{m}$ is a union of $\mathcal{E}_{m, i}$ for all $i \in\left\{1,2, \ldots, \frac{N}{K+1}-1\right\}$, or $\mathcal{E}_{m}=\bigcup_{k=1}^{\frac{N}{K+1}-1} \mathcal{E}_{m, i}$. Probability of error in the MERGE subroutine is given as

$$
\begin{aligned}
P\left(\mathcal{E}_{m}\right) & =P\left(\bigcup_{k=1}^{\frac{N}{K+1}-1} \mathcal{E}_{m, i}\right) \\
& \leq \sum_{k=1}^{\frac{N}{K+1}-1} P\left(\mathcal{E}_{m, i}\right) \\
& <\sum_{k=1}^{\frac{N}{K+1}-1} \frac{K}{2 N^{2} T^{2}} \\
& <\frac{N}{K+1} \frac{K}{2 N^{2} T^{2}} \\
& <\frac{1}{2 N T^{2}}
\end{aligned}
$$

where (63) and (64) follow from the definition of events and union bound, respectively, and (65) follows from Lemma 4.2.

Substituting bounds obtained on $P\left(\mathcal{E}_{m}\right)$ and $P\left(\mathcal{E}_{s}\right)$ in (56), we have

$$
\begin{aligned}
P(\mathcal{E}) & <\frac{1}{2 N T^{2}}+\frac{1}{2 N T^{2}} \\
& =\frac{1}{N T^{2}}
\end{aligned}
$$

The total error probability of the algorithm is thus bounded by $\frac{1}{N T^{2}}$, proving the statement of the Lemma.

\section{E PROOF OF LEMMA 4.4}

Exploration in Algorithm 1 is done using the SORT and MERGE subroutines, so we will analyze the maximum time taken by the two subroutines. To sort all groups, Algorithm 1 runs SORT $\frac{N}{K+1}$ times, and to merge the groups, Algorithm 1 runs $\frac{N}{K+1}-1$ times. Let $i^{t h}$ run of SORT uses $T_{S O R T, i}$ time steps, and $j^{t h}$ run of MERGE uses $T_{M E R G E, j}$ time steps. The total number of time-steps used for exploration can be written as

$$
\begin{aligned}
T_{\text {exp }}= & \sum_{s=1}^{\frac{N}{K+1}} T_{S O R T, s}+\sum_{m=1}^{\frac{N}{K+1}-1} T_{\text {MERGE, } m} \\
\leq & \sum_{s=1}^{\frac{N}{K+1}} \max _{s}\left(T_{S O R T, s}\right)+\sum_{m=1}^{\frac{N}{K+1}-1} \max _{m}\left(T_{M E R G E, m}\right) \\
= & \frac{N}{K+1} \max _{s}\left(T_{S O R T, s}\right) \\
& +\left(\frac{N}{K+1}-1\right) \max _{m}\left(T_{M E R G E, m}\right)
\end{aligned}
$$


We use Lemma 4.1 to find $\max _{S}\left(T_{S O R T, s}\right)$ as follows.

$$
\begin{aligned}
& \sum_{j=1}^{T_{S O R T, s}} \frac{64 U^{2} \log 2 N T}{\left(\max \left(\lambda, \delta_{s, j}\right)\right)^{2}} \\
\leq & \sum_{j, \delta_{s, j}>\lambda}^{K+1} \frac{64 U^{2} \log 2 N T}{\lambda^{2}}+\sum_{j, \delta_{s, j}<\lambda}^{K+1} \frac{64 U^{2} \log 2 N T}{\lambda^{2}} \\
= & \sum_{j=1}^{K+1} \frac{64 U^{2} \log 2 N T}{\lambda^{2}} \\
= & \frac{64(K+1) U^{2} \log 2 N T}{\lambda^{2}} .
\end{aligned}
$$

Similarly, we use Lemma 4.2 to find $\max _{s}\left(T_{M E R G E_{s}}\right)$ as follows.

$$
\begin{aligned}
& \sum_{j=1}^{T_{M E R G E, m}} \frac{64 U^{2} \log 2 N T}{\left(\max \left(\lambda, \delta_{m, j}\right)\right)^{2}} \\
\leq & \sum_{j, \delta_{m, j}>\lambda}^{K+1} \frac{64 U^{2} \log 2 N T}{\lambda^{2}}+\sum_{j, \delta_{m, j}<\lambda}^{K+1} \frac{64 U^{2} \log 2 N T}{\lambda^{2}} \\
= & \sum_{j=1}^{K+1} \frac{64 U^{2} \log 2 N T}{\lambda^{2}} \\
= & \frac{64(K+1) U^{2} \log 2 N T}{\lambda^{2}} .
\end{aligned}
$$

Total exploration time can now be bounded by using the values for maximum time taken for SORT and MERGE as,

$$
\begin{aligned}
& T_{\exp } \\
\leq & \frac{N}{K+1} \max _{s}\left(T_{\text {SORT }, s}\right)+\left(\frac{N}{K+1}-1\right) \max _{s}\left(T_{\text {SORT }, s}\right) \\
\leq & \frac{N}{K+1} \frac{64(K+1) U^{2} \log 2 N T}{\lambda^{2}} \\
& +\left(\frac{N}{K+1}-1\right) \frac{64(K+1) U^{2} \log 2 N T}{\lambda^{2}} \\
< & \frac{N}{K+1} \frac{64(K+1) U^{2} \log 2 N T}{\lambda^{2}} \\
& +\left(\frac{N}{K+1}\right) \frac{64(K+1) U^{2} \log 2 N T}{\lambda^{2}} \\
\leq & \frac{64 N U^{2} \log 2 N T}{\lambda^{2}}+\frac{64 N U^{2} \log 2 N T}{\lambda^{2}} \\
\leq & \frac{128 N U^{2} \log 2 N T}{\lambda^{2}} .
\end{aligned}
$$




\section{F PROOF OF LEMMA 4.5}

We will bound the expected regret where the suboptimal action is returned by CMAB-SM, which includes an error from Algorithm 2 or from Algorithm 3. Let the chosen action be $\hat{\mathbf{a}}^{*}=\left(\hat{a}_{1}^{*}\right.$, $\left.\ldots, \hat{a}_{K}^{*}\right)$ and optimal action be $\mathbf{a}^{*}=\left(a_{1}^{*}, \ldots, a_{K}^{*}\right)$. Then, the gap in the actions is $P=$ $\sqrt{\min _{\Pi}\left(\sum_{i=1}^{K}\left(\mathbf{d}_{\hat{a}_{i}^{*}}-\mathbf{d}_{a_{\Pi(i)}^{*}}\right)\right)^{2}}$, where $\Pi$ is any possible permutation of $\{1, \ldots, K\}$. Also, let $\Pi^{*}$ be the permutation that optimizes the above minimization. Using Assumption 4, and $U$ defined in Corollary 2, we have

$$
\mathbb{E}\left[r_{\mathrm{a}^{*}}-r_{\hat{\mathrm{a}}^{*}}\right] \leq U P
$$

We consider two possible cases. Case 1 corresponds to the scenario where for some $i, \mathbb{E}\left[\mathbf{d}_{\hat{a}_{i}^{*}}\right]-$ $\mathbb{E}\left[\mathbf{d}_{a_{\Pi^{*}(i)}^{*}}\right]>\lambda$. The second case is when for all $i, \mathbb{E}\left[\mathbf{d}_{\hat{a}_{i}^{*}}\right]-\mathbb{E}\left[\mathbf{d}_{a_{\Pi^{*}(i)}^{*}}\right] \leq \lambda$.

Case 1: For some $i, \mathbb{E}\left[\mathbf{d}_{\hat{a}_{i}^{*}}\right]-\mathbb{E}\left[\mathbf{d}_{a_{\Pi^{*}(i)}^{*}}\right]>\lambda$. In this case, the incorrect action has arms which could have been separated without hitting the threshold $\lambda$, but did not do so because of an error in the SORT or MERGE subroutine. We note that since $\mathbb{E}\left[\mathbf{d}_{\hat{a}_{i}^{*}}\right]$ and $\mathbb{E}\left[\mathbf{d}_{a_{\Pi^{*}(i)}^{*}}\right]$ are both in $[0,1], P \leq \sqrt{K}$. The expected regret at time $t$ can be written as

$$
\begin{aligned}
\mathbb{E}[R(t)] & =\mathbb{E}\left[r_{\mathbf{a}^{*}}-r_{\hat{\mathbf{a}}^{*}} \mid \mathbf{a}_{t} \neq \mathbf{a}^{*}\right] \times P\left(\left\{\mathbf{a}_{t} \neq \mathbf{a}^{*}\right\}\right) \\
& \leq U \sqrt{K} \times P\left(\left\{\mathbf{a}_{t} \neq \mathbf{a}^{*}\right\}\right) \\
& \leq \frac{U \sqrt{K}}{N T^{2}}
\end{aligned}
$$

where (72) follows from (70) with $P \leq \sqrt{K}$, and (73) follows from Lemma 4.3 .

Case 2: For all $i, \mathbb{E}\left[\mathbf{d}_{\hat{a}_{i}^{*}}\right]-\mathbb{E}\left[\mathbf{d}_{a_{\Pi^{*}(i)}^{*}}\right] \leq \lambda$. In this case, the SORT or MERGE subroutines will not be able to differentiate between the two actions. Thus, $P\left(\left\{\mathbf{a}_{t} \neq \mathbf{a}^{*}\right\}\right)$ will no longer be bounded by $1 / N T^{2}$ in this case, since the individual rewards are bounded by $\lambda$, we have $P \leq \lambda \sqrt{K}$. Thus, we have

$$
\begin{aligned}
\mathbb{E}[R(t)] & =\mathbb{E}\left[r_{\mathbf{a}^{*}}-r_{\hat{a}^{*}} \mid \mathbf{a}_{t} \neq \mathbf{a}^{*}\right] \times P\left(\left\{\mathbf{a}_{t} \neq \mathbf{a}^{*}\right\}\right) \\
& \leq U \lambda \sqrt{K} \times P\left(\left\{\mathbf{a}_{t} \neq \mathbf{a}^{*}\right\}\right) \\
& \leq U \lambda \sqrt{K},
\end{aligned}
$$

where (75) follows from (70) and $P \leq \lambda \sqrt{K}$.

Combining both the cases, the maximum regret the algorithm can incur is bounded by $U \lambda \sqrt{K}+$ $\frac{U \sqrt{K}}{N T^{2}}$, thus proving the result.

\section{G COMPLETE IMPLEMENTATION OF SORT AND MERGE SUBROUTINES}

The detailed SORT and MERGE subroutines can be seen in Algorithm 4-6.

\section{H SYNTHETIC EVALUATION RESULTS FOR BERNOULLI REWARDS}

In this section, we evaluate CMAB-SM under multiple synthetic problem settings. We compare the result with the improved UCB algorithm as described in [6]. Since this paper provides the first result with non-linear reward functions for the $\mathrm{CMAB}$ problem with bandit feedback, we compare with the UCB algorithm [6] which is optimal for small $N$ and $K$ while having the regret scale with $\left(\begin{array}{l}N \\ K\end{array}\right)$.

For evaluations, we ran the algorithm for $T=10^{6}$ time steps and averaged over 30 runs. We compare cumulative regret at each $t$ starting from $t=0$, which is defined as, 


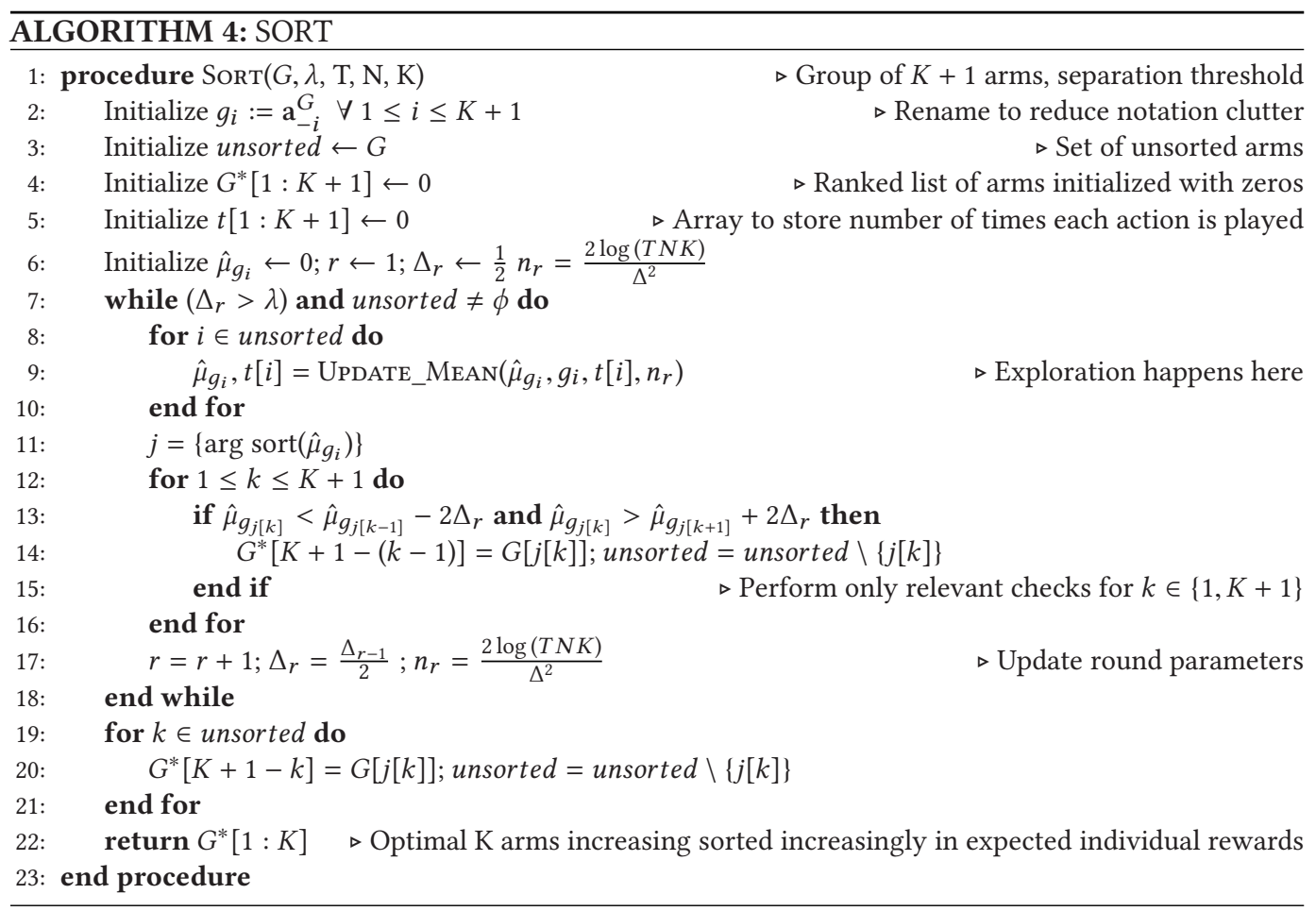

$$
W(t)=\sum_{t^{\prime}=0}^{t} R\left(t^{\prime}\right)
$$

We consider two values of $N \in\{12,24\}$. For $N=12$, we choose $K \in\{2,3,5\}$, while for $N=24$, we choose $K \in\{2,3,5,7,11\}$. Since the arms must have FSD over each other, we describe an example single parameter distribution for the reward of each arm that has this property. Reward of arm $i$ comes from the set $\{0,1\}$ and follows a Bernoulli distribution with parameter $p_{i}$, or

$$
X_{i} \sim \operatorname{Bern}\left(p_{i}\right) .
$$

We note that arm $i$ has FSD over $\operatorname{arm} j$ if $p_{i}>p_{j}$. Thus, this reward distribution satisfies Assumption 2 as long as no two arms have the same parameter, or $p_{i} \neq p_{j}$ for any $i \neq j$. Figure $3(\mathrm{~b})$ plots $P(X \geq x)$ of the reward function for different values of $p_{i}$. We see that $P(X \geq x)$ is larger for the distribution with larger value of $p$, and for any two different values of $p$, there is $x$ (e.g., any $x \in(0,1])$ such that $P(X \geq x)$ are not the same thus showing that the reward distributions satisfy Assumption 2.

We consider two different types of reward functions to evaluate the performance of CMAB-SM. The two reward functions are the sum of rewards of each arm, and the maximum of rewards of each arm, In the following, we will describe the functions and evaluate them for one of the distributions of the rewards from each arm.

\section{H.1 Sum of Bernoulli Arm Rewards}

Assume that a company wishes to do a campaign of a product and chooses $K$ out of available $N$ subcampaigns each day. Let the reward of sub-campaign $i, X_{i}$, be Bernoulli with parameter $p_{i}$, which is unknown. Further, let the reward that the company receives is how the overall company sales 

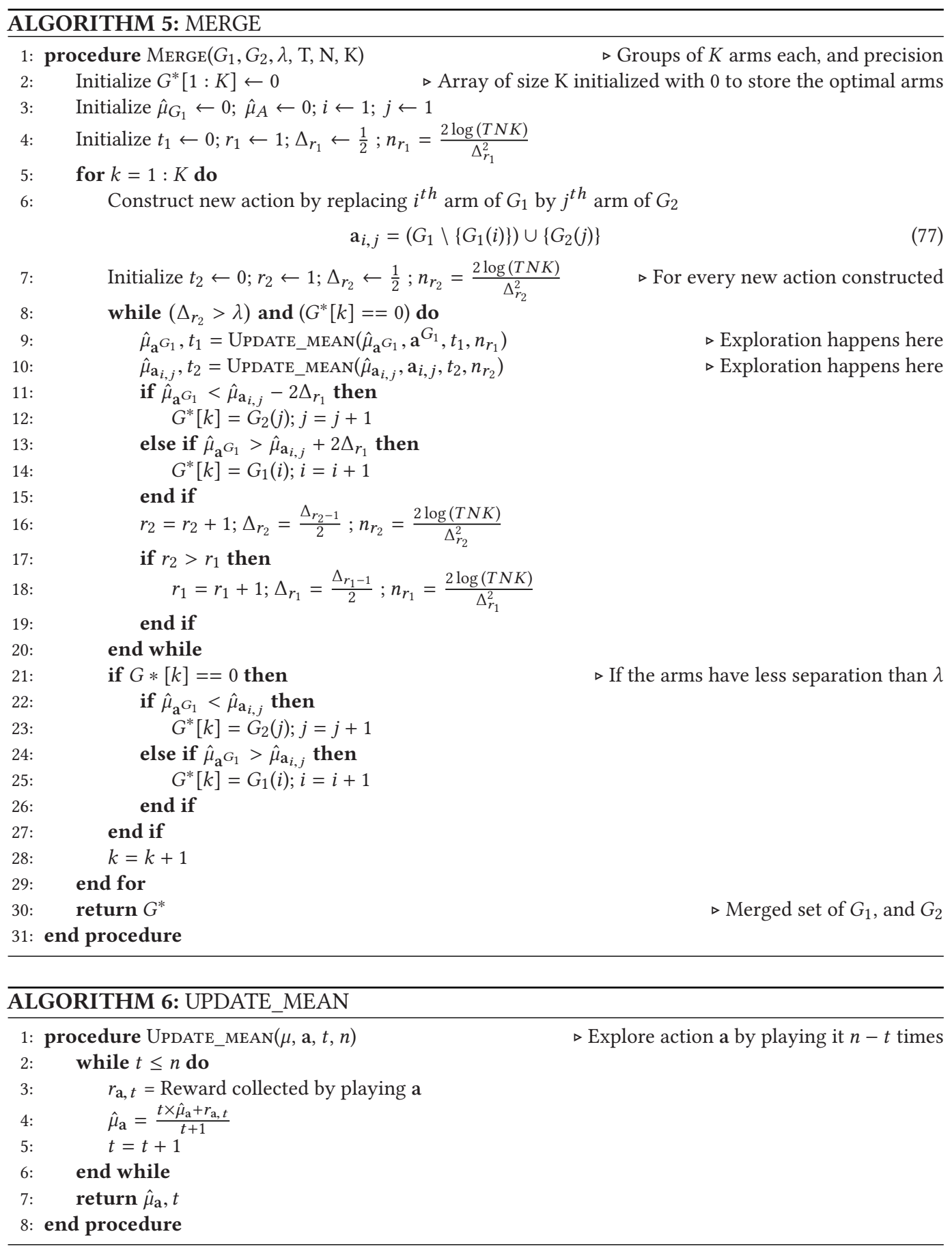

progressed, thus receiving the aggregate reward as $\sum_{i=1}^{K} X_{i}$. To normalize the received reward, we let $r=\frac{1}{K} \sum_{i=1}^{K} X_{i}$. We assume that the individual arm rewards are not observed by the company, while the overall progress of the campaign can be seen. Another application is showing $K$ out of $N$ advertisements to the user webpage, where the reward is in a form of whether the user clicks the 


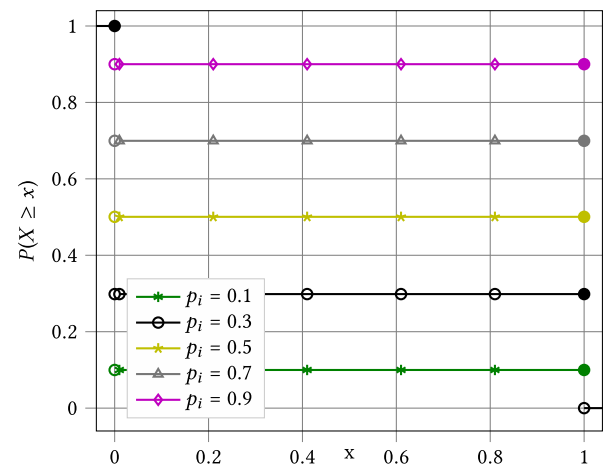

(a) Bernoulli Rewards

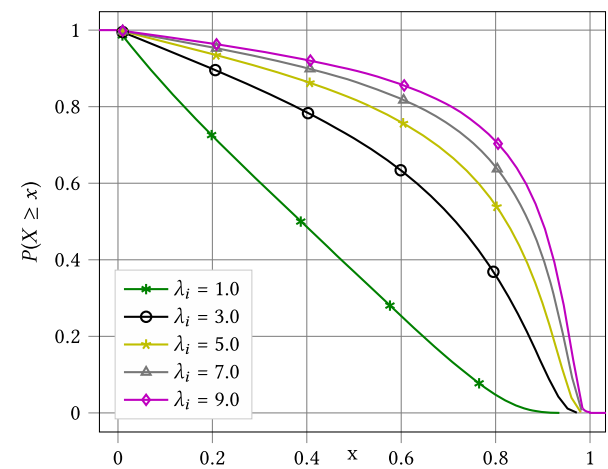

(b) Transformed Rewards

Fig. 3. First Order Stochastic Dominance on reward distribution.

ad to go to the product page. The aggregate reward is the total number of clicks, and we assume that individual click information is not available.

Thus, we have the expected reward as

$$
\begin{aligned}
\mathbb{E}[r] & =\mathbb{E}\left[\frac{1}{K} \sum_{i=1}^{K} X_{i}\right] \\
& =\frac{1}{K} \sum_{i=1}^{K} \mathbb{E}\left[X_{i}\right] \\
& =\frac{1}{K} \sum_{i=1}^{K} p_{i}
\end{aligned}
$$

Since the combined reward is the sum of all individual rewards, the expected reward is strictly increasing with respect to the expected rewards of the individual arms. Prior works in click optimization assume knowledge of clicks on individual advertisements and thus are semi-bandits [25].

Recently, [37] proposed a CSAR algorithm to find top $K$ arms which solves this problem setup efficiently using Hadamard matrices. The CSAR algorithm, similar to ours, divides the set of arms into groups of size $2 K$. The algorithm then estimates the individual arm rewards of the $2 K$ arms in each group using Hadamard matrices of size $2 K$. Each column maps to an arm in the group and for each row, an action can now be constructed by selecting all arms corresponding to +1 elements in the row or the arms corresponding to -1 elements in the row.

We compare our CMAB-SM with the UCB algorithm and CSAR algorithm for $N=45$ and various values of $K$ for $T=10^{6}$ steps. For the CSAR algorithm we select $K \in\{2,4,8\}$ for easy construction of Hadamard matrices of size $2 K$. For the UCB algorithm we select $K \in\{2,4\}$ for easy construction of the combinatorial action space of $\left(\begin{array}{l}N \\ K\end{array}\right)$. We plot average cumulative regret over 25 independent runs for a fixed value of individual arm rewards sampled randomly uniformly from $[0,1]$.

Figure 4 shows the comparison results between CMAB-SM, CSAR algorithm, and UCB algorithm. We note that for $K=2$, UCB algorithm outperforms both CSAR algorithm and CMAB-SM algorithm. This is because for small values of $K, \mathrm{UCB}$ can explore all $\left(\begin{array}{l}N \\ K\end{array}\right)$ actions faster as UCB does not estimate individual arm rewards as does the CSAR algorithm, and UCB can eliminate sub optimal arms from direct comparison with the best arm unlike CMAB-SM algorithm. We note that in the cumulative regret of CSAR algorithm rises sharply for $K=2$ in Figure 4(a). We suspect that this is because of a large number of samples might be required to eliminate a sub-optimal arm. 


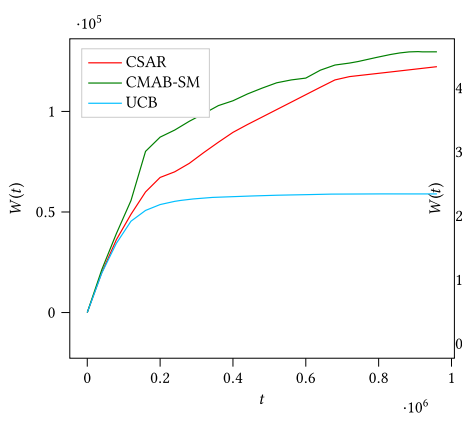

(a) $K=2$

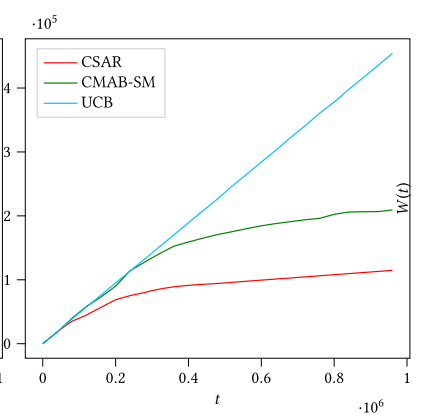

(b) $K=4$

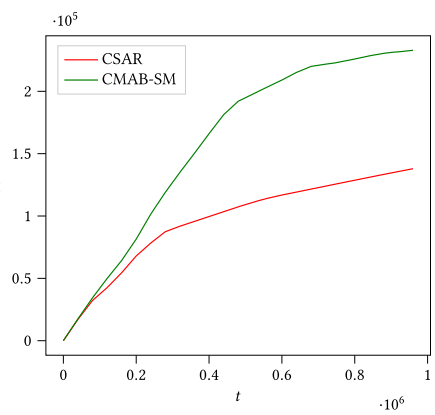

(c) $K=8$

Fig. 4. Comparison results for CMAB-SM and CSAR Algorithm for combinatorial linear bandit reward as the mean of individual arm rewards.

Since our implementation of the algorithm samples a group consecutively for $m=1 / 2^{r}$ times in round $r$, we see a jump instead of a smooth rise. Such behaviour is not visible for $K=4$ or $K=8$ because we suspect that the elimination of sub-optimal arms might have been quicker compared to the case of $K=2$ because of the large gap between arms.

Also, we note that CMAB-SM does not perform as good as the CSAR algorithm. We note that this difference is because CSAR estimates expected rewards of individual arms. CSAR algorithm can construct optimal action from the estimates and pull the optimal arm more frequently. CMABSM eliminates arms slowly compared to CSAR algorithm, as CSAR compares each arm from the $K^{\text {th }}$ best arm which CMAB cannot perform. However, we note that even for $K=4$, both CSAR and CMAB-SM algorithm outperform UCB algorithm by a significant margin. Also, for $K=8$, we note that UCB algorithm would not even finish exploring $\left(\begin{array}{c}45 \\ 8\end{array}\right)=215553195$ arms in $10^{6}$ time steps. Hence, a comparison with UCB for $K=8$ is futile.

\section{H.2 Maximum of Bernoulli Rewards}

We consider a case where agent is a recommendation system that shows a list of restaurants or hotels, and user provides feedback whether or not the list is useful. A user finds the list useful when she is able to get a recommendation suiting her requirements. We take the reward of individual arm to be discrete with value 1 if the item was useful, and 0 for the case where the item in the list is not useful. We assume that the rewards follow Bernoulli distribution. Since the individual rewards are not observed, this is a bandit setting. Further, note that the maximum function is not a linear function. We will now show the strictly increasing property of the function. The expected reward of selecting $K$ arms is given as

$$
\begin{aligned}
\mathbb{E}[r] & =\mathbb{E}\left[\max \left(X_{1}, X_{2}, \ldots, X_{K}\right)\right] \\
& =1\left(1-P\left(\bigcap_{i=1}^{K}\left\{X_{i}=0\right\}\right)\right)+0\left(P\left(\bigcap_{i=1}^{K}\left\{X_{i}=0\right\}\right)\right) \\
& =1\left(1-\prod_{i=1}^{K}\left(1-p_{i}\right)\right)+0\left(\prod_{i=1}^{K}\left(1-p_{i}\right)\right) \\
& =1-\prod_{i=1}^{K}\left(1-p_{i}\right),
\end{aligned}
$$




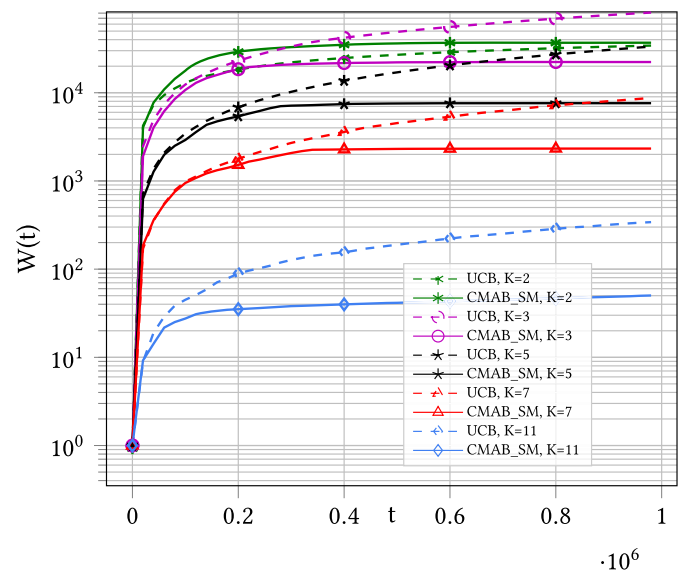

(a) $\mathrm{N}=24$

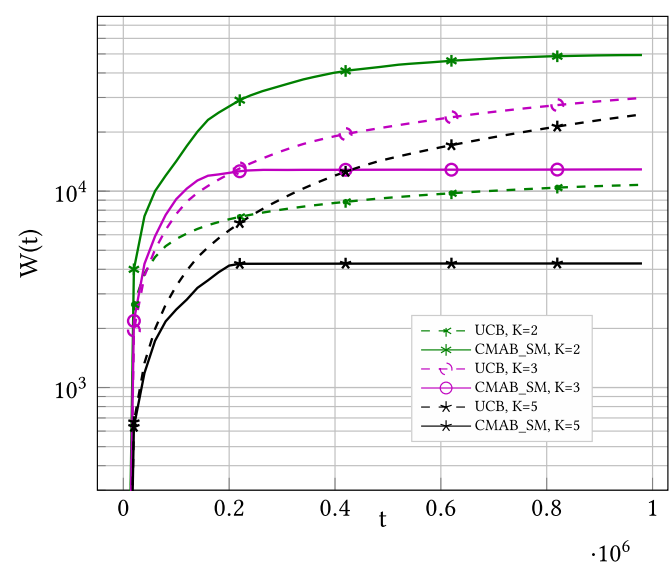

(b) $\mathrm{N}=12$

Fig. 5. Reward of actions is the maximum of rewards of individual arms as described in section H.2.

where (78) is the expected value of the function of individual rewards, (79) follows from the fact that individual rewards are Bernoulli distributed and their maximum is zero only when all the individual rewards are zero, and (80) holds since the rewards are independent of each other.

[18] present a unique way to solve this problem in Section 4.2 of of their paper. They consider the success probability $p_{i}$ of arm $i$ comes from the set $\left\{1-\beta^{R}, 1-\beta^{R-1}, \ldots, 1-\beta\right\}$ for all $i \in\{1,2, \ldots, N\}$ and $\beta \in(0,1)$ and $R>0$ are fixed parameters. Additionally, their bounds are of order $O\left(\left(\begin{array}{c}N-1 \\ K\end{array}\right)\right)$. Our setting is a generalization of the setting considered by [18]. We let the $p_{i}$ lie in the set $[0,1]$ and we obtain a bound which is polynomial in $N, K$.

The reward is non-linear and the expected value of reward is strictly increasing in expected rewards of individual arms. Cascade model of click optimization by [26] uses a similar problem formulation, however they still consider information on clicks on individual items. Figure 5 shows the evaluation results in this case. We note that for $K \geq 3$, the proposed algorithm significantly outperforms UCB. Even for $N=24$ and $K=2$, where $\left(\begin{array}{l}N \\ K\end{array}\right)=220, W(T)$ for CMAB-SM is close to UCB.

We note that the cumulative regret at any time $t$ decreases as $K$ increases. This follows from the fact that as with increasing $K$, a user will have more choices at any given time and it is more likely that the arm with the highest reward is in the $K$ chosen arms.

\section{H.3 Comparisons for Linear Approximation of Rewards}

We also compare the proposed CMAB-SM algorithm with the LinUCB algorithm [32]. The LinUCB algorithm models the joint reward as linear combination of individual arm rewards. The total action space for the LinUCB algorithm becomes $\left(\begin{array}{l}N \\ K\end{array}\right)$ which makes it inefficient for storage. Also, the time complexity of the LinUCB algorithm becomes $O\left(N^{3}\left(\begin{array}{l}N \\ K\end{array}\right)\right)$ as it requires matrix multiplication of $\left(\begin{array}{l}N \\ K\end{array}\right) \times N$ size matrices to compute UCB values for every interaction with the environment. We plot the regret for setup where the joint reward is a quadratic function of individual rewards in Figure 6. We note that for small values of $K$, the UCB algorithm incurs a lower regret compared to the CMAB-SM algorithm. However, as $K$ increases, the number of actions increase and the regret of the UCB algorithm also increases. 


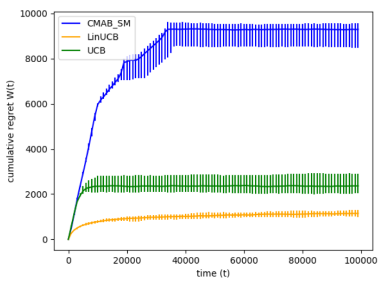

(a) $K=2$

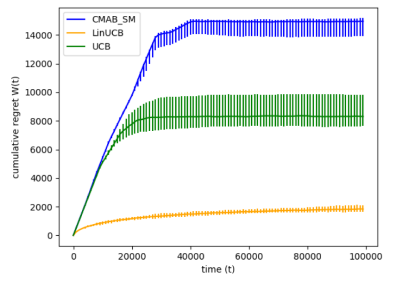

(b) $K=3$

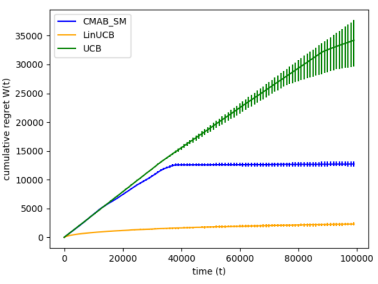

(c) $K=5$

Fig. 6. Regret growth for the CMAB-SM, UCB, and LinUCB algorithm for environment where the joint reward is a quadratic function of individual arm rewards.

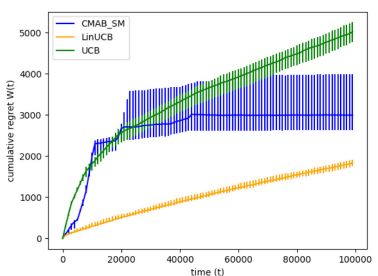

(a) $K=2$

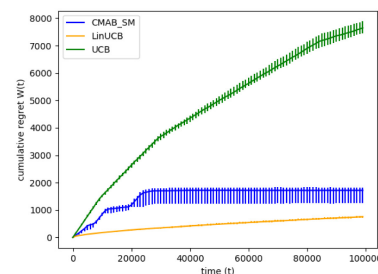

(b) $K=3$

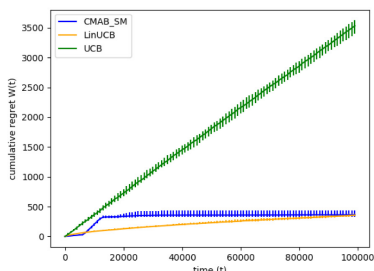

(c) $K=5$

Fig. 7. Regret growth for the CMAB-SM, UCB, and LinUCB algorithm for environment where the joint reward is the maximum of individual arm rewards. The regret growth of LinUCB algorithm becomes comparable to the proposed CMAB-SM algorithm as $K$ increases along with a significant increase in computational costs.

For the setup where the joint reward is the maximum of rewards of individual arms, we plot the regrets in Figure 7. The UCB algorithm performs significantly worse than the LinUCB algorithm. Also, the regret of the LinUCB algorithm starts becoming higher than the regret of the CMAB-SM algorithm because of the non-linearity of the function. Further, we note that at every time step, the LinUCB algorithm requires $\left(\begin{array}{c}12 \\ 3\end{array}\right) \times 1000$ times more computations than the CMAB-SM algorithm.

\section{ASSUMPTIONS FOR SOCIAL INFLUENCE MAXIMIZATION}

We note that although several assumptions do not strictly hold for the problem of social influence maximization, related properties do. These are:

(1) Assumption 1 of symmetry: The reward function for the social influence maximization problem is independent of the seed set ordering.

(2) Assumption 4: The social influence maximization objective function is a submodular set function [23], which implies bounds similar to Lipschitz Continuity.

(3) Assumptions 3 and 5 of strictly increasing and continuous reward function: In the social network, if the influence of a single node increases slightly, such as through increased edge weights, the expected total reward of any seed set containing that node will most often increase, but not drastically so.

The only situation in which there will be an increase for individual node rewards but no increase in the expected total reward is when only weights on edges between nodes in the seed set are increased.

Increasing edge weights (and consequently individual node rewards) will never decrease the expected total reward for any seed set. 


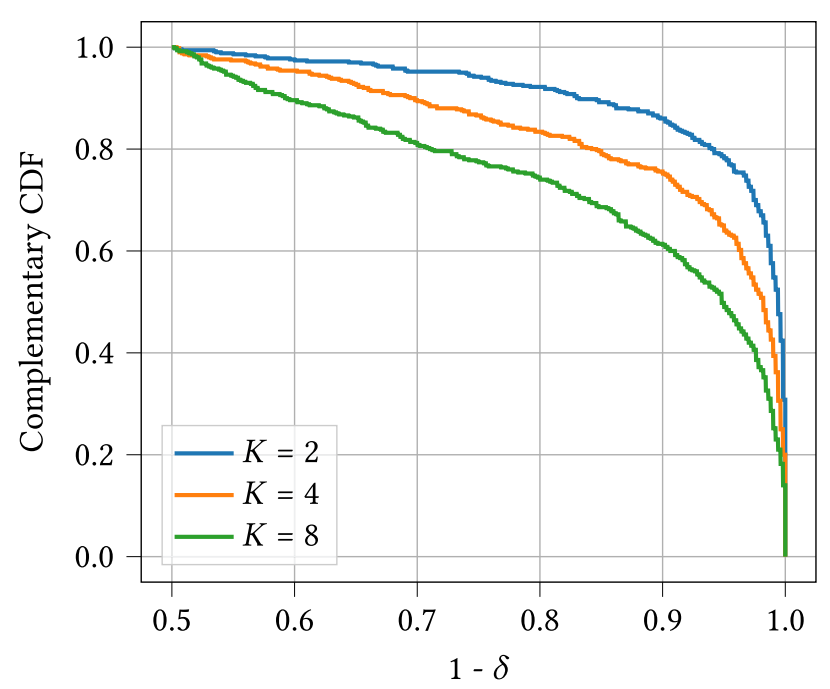

Fig. 8. Complementary CDF of the approximate dominance in expectation probabilities $1-\delta$ for different values of $K$ for the Facebook friends network. The height of each curve indicates what fraction of nodes satisfy this weaker form of dominance.

(4) Stochastic dominance does not hold in general for the problem of social influence maximization. However, we empirically found that a weaker version of stochastic dominance holds with non-negligible probability in the network we studied. We now explain this weaker version of stochastic dominance in detail.

In Section 6.2, we discussed how influence maximization does not (strictly) satisfy the assumptions of stochastic dominance of individual arms or the reward being a (strictly increasing) function of individual arm rewards. However, we have empirically found evidence that a related property holds. We now introduce this related property, a weaker form of stochastic dominance for size $K$ sets.

Let $a, b \in[N]$ denote two distinct arm indices. Let $S_{a, b} \in([N] \backslash\{a, b\})^{K-1}$ denote a random set of $K-1$ distinct arm indices, uniformly distributed over all $\left(\begin{array}{c}N-2 \\ K-1\end{array}\right)$ subsets of $[N] \backslash\{a, b\}$ with cardinality $K-1$.

Definition. Approximate Dominance in Expectation. Arm $a$ is said to approximately dominate $\operatorname{arm} b$ in expectation, with probability $1-\delta$, if

$$
\begin{aligned}
\operatorname{Prob}[ & \mathrm{E}_{P_{\mathrm{X}}}\left[f\left(X_{S_{a, b}(1)}, \ldots, X_{S_{a, b}(K-1)}, X_{a}\right)\right] \\
& \left.\geq \mathrm{E}_{P_{\mathrm{X}}}\left[f\left(X_{S_{a, b}(1)}, \ldots, X_{S_{a, b}(K-1)}, X_{b}\right)\right]\right]=1-\delta,
\end{aligned}
$$

where the inner expectations are with respect to the arm distributions and the outer probability is taken with respect to the the uniform measure over possible $S_{a, b}$.

Stochastic dominance, with a symmetric and strictly increasing reward function $f(\cdot)$, implies this dominance in expectation with $\delta=0$. The converse is not true.

We empirically assessed the prevalence of this property for the influence maximization problem.

For each pair of nodes $a$ and $b$ (w.l.o.g. ordered so $a$ approximately dominates $b$ in expectation), there is a corresponding $1-\delta \geq 0.5$. We uniformly sampled from pairs $\{a, b\}$ at random, inducing a probability distribution on the $\delta$ values. 
For each $K=2,4,8$, for 500 different pairs of $\{a, b\}$, we calculated the above probability over a uniform distribution over $S_{a, b}$ (based on 500 random samples from $S_{a, b}$ ), where the expected influence of any set was calculated as a sample average based on 500 diffusions. We observed that the probabilistic stochastic dominance is satisfied with a reasonably high probability.

We computed the complementary cumulative distribution of $1-\delta$ to indicate what fraction of pairs of nodes $\{a, b\} \subset[N]$ (such that either $a$ or $b$ is in the optimal set for a given $K$ ) satisfy approximate stochastic dominance with a probability $1-\delta$ of at least a given value.

Figure 8 displays the empirical complementary cumulative distribution function of $1-\delta$ values for different values of $K$ from Facebook friends network [31]. If stochastic dominance held, then the curves would be flat with a value of 1 . Thus, higher values indicate stronger dominance. $K=2$ has the highest values, then $K=4$ and $K=8$, respectively. Thus, even for $K=8$, for example, about half of all pairs of nodes $\{a, b\} \subset[N]$ satisfy approximate dominance in expectation with a probability of at least 0.9 .

\section{REFERENCES}

[1] Yasin Abbasi-Yadkori, David Pal, and Csaba Szepesvari. 2011. Improved algorithms for linear stochastic bandits. In Advances in Neural Information Processing Systems 24. 2312-2320.

[2] Mridul Agarwal and Vaneet Aggarwal. 2018. Regret bounds for stochastic combinatorial multi-armed bandits with linear space complexity. arXiv preprint arXiv:1811.11925 (2018).

[3] Mridul Agarwal, Vaneet Aggarwal, Christopher J. Quinn, and Abhishek Umrawal. 2021. DART: aDaptive accept reject for non-linear top-k subset identification. In Proc. AAAI.

[4] Mridul Agarwal, Vaneet Aggarwal, Christopher J. Quinn, and Abhishek K. Umrawal. 2021. Stochastic Top-K subset bandits with linear space and non-linear feedback. In Algorithmic Learning Theory. PMLR, 306-339.

[5] Jean-Yves Audibert, Sébastien Bubeck, and Gábor Lugosi. 2014. Regret in online combinatorial optimization. Math. Oper. Res. 39, 1 (Feb. 2014), 31-45. https://doi.org/10.1287/moor.2013.0598

[6] Peter Auer and Ronald Ortner. 2010. UCB revisited: Improved regret bounds for the stochastic multi-armed bandit problem. Periodica Mathematica Hungarica 61, 1-2 (2010), 55-65.

[7] Vijay S. Bawa. 1975. Optimal rules for ordering uncertain prospects. fournal of Financial Economics 2, 1 (1975), 95-121.

[8] Lilian Besson and Emilie Kaufmann. 2018. What doubling tricks can and can't do for multi-armed bandits. arXiv.org (2018).

[9] Vincent D. Blondel, Jean-Loup Guillaume, Renaud Lambiotte, and Etienne Lefebvre. 2008. Fast unfolding of communities in large networks. fournal of Statistical Mechanics: Theory and Experiment 2008, 10 (2008), P10008.

[10] Nicolò Cesa-Bianchi and Gábor Lugosi. 2012. Combinatorial bandits. f. Comput. Syst. Sci. 78, 5 (Sept. 2012), $1404-1422$. https://doi.org/10.1016/j.jcss.2012.01.001

[11] Wei Chen, Yajun Wang, and Yang Yuan. 2013. Combinatorial multi-armed bandit: General framework and applications. In Proceedings of the 30th International Conference on Machine Learning (Proceedings of Machine Learning Research, Vol. 28), Sanjoy Dasgupta and David McAllester (Eds.). PMLR, Atlanta, Georgia, USA, 151-159. http://proceedings.mlr. press/v28/chen13a.html.

[12] Varsha Dani, Thomas Hayes, and Sham Kakade. 2008. Stochastic linear optimization under bandit feedback. In Proceedings of the 21st Annual Conference on Learning Theory. 355-366.

[13] Varsha Dani, Thomas P. Hayes, and Sham M. Kakade. 2008. Stochastic linear optimization under bandit feedback. In 21st Annual Conference on Learning Theory - COLT 2008, Helsinki, Finland, fuly 9-12, 2008, Rocco A. Servedio and Tong Zhang (Eds.). Omnipress, 355-366. http://colt2008.cs.helsinki.fi/papers/80-Dani.pdf.

[14] Varsha Dani, Sham M. Kakade, and Thomas P. Hayes. 2008. The price of bandit information for online optimization. In Advances in Neural Information Processing Systems 20, J. C. Platt, D. Koller, Y. Singer, and S. T. Roweis (Eds.). Curran Associates, Inc., 345-352.

[15] Pedro Domingos and Matt Richardson. 2001. Mining the network value of customers. In Proceedings of the Seventh ACM SIGKDD International Conference on Knowledge Discovery and Data Mining. ACM, 57-66.

[16] Sarah Filippi, Olivier Cappe, Aurelien Garivier, and Csaba Szepesvari. 2010. Parametric bandits: The generalized linear case. In Advances in Neural Information Processing Systems 23. 586-594.

[17] Yi Gai, Bhaskar Krishnamachari, and Rahul Jain. 2010. Learning multiuser channel allocations in cognitive radio networks: A combinatorial multi-armed bandit formulation. In New Frontiers in Dynamic Spectrum, 2010 IEEE Symposium on. IEEE, 1-9. 
[18] Aditya Gopalan, Shie Mannor, and Yishay Mansour. 2014. Thompson sampling for complex online problems. In Proceedings of the 31st International Conference on Machine Learning. 100-108.

[19] Amit Goyal. 2011. Credit Distribution Source Code Release. https://www.cs.ubc.ca/ goyal/code-release.php. Accessed: 2019-04-10.

[20] Amit Goyal, Francesco Bonchi, and Laks VS Lakshmanan. 2011. A data-based approach to social influence maximization. Proceedings of the VLDB Endowment 5, 1 (2011), 73-84.

[21] Josef Hadar and William R. Russell. 1969. Rules for ordering uncertain prospects. The American Economic Review 59, 1 (1969), 25-34.

[22] Kwang-Sung Jun, Aniruddha Bhargava, Robert Nowak, and Rebecca Willett. 2017. Scalable generalized linear bandits: Online computation and hashing. In Advances in Neural Information Processing Systems 30. 98-108.

[23] David Kempe, Jon Kleinberg, and Éva Tardos. 2003. Maximizing the spread of influence through a social network. In Proceedings of the Ninth ACM SIGKDD International Conference on Knowledge Discovery and Data Mining. ACM, 137-146.

[24] O. Krafft and N. Schmitz. 1969. A note on Hoeffding's Inequality. J. Amer. Statist. Assoc. 64, 327 (1969), $907-912$.

[25] Branislav Kveton, Chang Li, Tor Lattimore, Ilya Markov, Maarten de Rijke, Csaba Szepesvari, and Masrour Zoghi. 2018. BubbleRank: Safe online learning to rerank. arXiv preprint arXiv:1806.05819 (2018).

[26] Branislav Kveton, Csaba Szepesvari, Zheng Wen, and Azin Ashkan. 2015. Cascading bandits: Learning to rank in the cascade model. In Proceedings of the 32nd International Conference on Machine Learning (Proceedings of Machine Learning Research, Vol. 37), Francis Bach and David Blei (Eds.). PMLR, Lille, France, 767-776. http://proceedings.mlr. press/v37/kveton15.html.

[27] Branislav Kveton, Zheng Wen, Azin Ashkan, Hoda Eydgahi, and Brian Eriksson. 2014. Matroid bandits: Fast combinatorial optimization with learning. arXiv preprint arXiv:1403.5045 (2014).

[28] Branislav Kveton, Zheng Wen, Azin Ashkan, and Csaba Szepesvari. 2015. Tight regret bounds for stochastic combinatorial semi-bandits. In Artificial Intelligence and Statistics. 535-543.

[29] Branislav Kveton, Manzil Zaheer, Csaba Szepesvari, Lihong Li, Mohammad Ghavamzadeh, and Craig Boutilier. 2020. Randomized exploration in generalized linear bandits. In 23rd International Conference on Artificial Intelligence and Statistics.

[30] Siyu Lei, Silviu Maniu, Luyi Mo, Reynold Cheng, and Pierre Senellart. 2015. Online influence maximization. In Proceedings of the 21th ACM SIGKDD International Conference on Knowledge Discovery and Data Mining. ACM, 645-654.

[31] Jure Leskovec and Julian J. Mcauley. 2012. Learning to discover social circles in ego networks. In Advances in Neural Information Processing Systems. 539-547.

[32] Lihong Li, Wei Chu, John Langford, and Robert E. Schapire. 2010. A contextual-bandit approach to personalized news article recommendation. In Proceedings of the 19th International Conference on World Wide Web. 661-670.

[33] Lihong Li, Yu Lu, and Dengyong Zhou. 2017. Provably optimal algorithms for generalized linear contextual bandits. In Proceedings of the 34th International Conference on Machine Learning. 2071-2080.

[34] David Liau, Zhao Song, Eric Price, and Ger Yang. 2018. Stochastic multi-armed bandits in constant space. In Proceedings of the Twenty-First International Conference on Artificial Intelligence and Statistics (Proceedings of Machine Learning Research, Vol. 84), Amos Storkey and Fernando Perez-Cruz (Eds.). PMLR, Playa Blanca, Lanzarote, Canary Islands, 386-394. http://proceedings.mlr.press/v84/liau18a.html.

[35] Tian Lin, Bruno Abrahao, Robert Kleinberg, John Lui, and Wei Chen. 2014. Combinatorial partial monitoring game with linear feedback and its applications. In Proceedings of the 31st International Conference on Machine Learning (Proceedings of Machine Learning Research, Vol. 32), Eric P. Xing and Tony Jebara (Eds.). PMLR, Bejing, China, 901-909. http://proceedings.mlr.press/v32/lind14.html.

[36] Alessandro Nuara, Francesco Trovo, Nicola Gatti, Marcello Restelli, et al. 2018. A combinatorial-bandit algorithm for the online joint bid/budget optimization of pay-per-click advertising campaigns. In Thirty-Second AAAI Conference on Artificial Intelligence. 1840-1846.

[37] Idan Rejwan and Yishay Mansour. 2020. Top- $k$ combinatorial bandits with full-bandit feedback. In Algorithmic Learning Theory. 752-776.

[38] Alan B. Slomson. 1997. Introduction to Combinatorics. CRC Press.

[39] Sharan Vaswani, Branislav Kveton, Zheng Wen, Mohammad Ghavamzadeh, Laks VS Lakshmanan, and Mark Schmidt. 2017. Model-independent online learning for influence maximization. In Proceedings of the 34th International Conference on Machine Learning-Volume 70. JMLR. org, 3530-3539.

[40] Zheng Wen, Branislav Kveton, Michal Valko, and Sharan Vaswani. 2016. Online influence maximization under independent cascade model with semi-bandit feedback. arXiv preprint arXiv:1605.06593 (2016).

[41] Zheng Wen, Branislav Kveton, Michal Valko, and Sharan Vaswani. 2017. Online influence maximization under independent cascade model with semi-bandit feedback. In Advances in Neural Information Processing Systems. 3022-3032. 
[42] Raymond Chi-Wing Wong, Ada Wai-Chee Fu, and K. Wang. 2003. MPIS: Maximal-profit item selection with crossselling considerations. In Third IEEE International Conference on Data Mining. 371-378.

[43] Yu Xiang, Tian Lan, Vaneet Aggarwal, and Yih-Farn R. Chen. 2016. Joint latency and cost optimization for erasurecoded data center storage. IEEE/ACM Transactions on Networking (TON) 24, 4 (2016), 2443-2457.

[44] Weinan Zhang, Ying Zhang, Bin Gao, Yong Yu, Xiaojie Yuan, and Tie-Yan Liu. 2012. Joint optimization of bid and budget allocation in sponsored search. In Proceedings of the 18th ACM SIGKDD International Conference on Knowledge Discovery and Data Mining. ACM, 1177-1185.

[45] Zhijie Zhang, Wei Chen, Xiaoming Sun, and Jialin Zhang. 2021. Online influence maximization with node-level feedback using standard offline oracles. arXiv preprint arXiv:2109.06077 (2021).

Received April 2021; revised November 2021; accepted December 2021 